\title{
Three-Axis Attitude Estimation Using Rate-Integrating Gyroscopes
}

\author{
John L. Crassidis* \\ University at Buffalo, State University of New York, Amherst, NY, 14260-4400 \\ F. Landis Markley ${ }^{\dagger}$ \\ NASA Goddard Space Flight Center, Greenbelt, Maryland 20771
}

\begin{abstract}
Traditionally, attitude estimation has been performed using a combination of external attitude sensors and internal three-axis gyroscopes. There are many studies of three-axis attitude estimation using gyros that read angular rates. Rate-integrating gyros measure integrated rates or angular displacements, but three-axis attitude estimation using these types of gyros has not been as fully investigated. This paper derives a Kalman filtering framework for attitude estimation using attitude sensors coupled with rateintegrating gyroscopes. In order to account for correlations introduced by using these gyros, the state vector must be augmented, compared with filters using traditional gyros that read angular rates. Two filters are derived in this paper. The first uses an augmented state-vector form that estimates attitude, gyro biases, and gyro angular displacements. The second ignores correlations, leading to a filter that estimates attitude and gyro biases only. Simulation comparisons are shown for both filters. The work presented in this paper focuses only on attitude estimation using rate-integrating gyros, but it can easily be extended to other applications such as inertial navigation, which estimates attitude and position.
\end{abstract}

\footnotetext{
${ }^{*}$ CUBRC Professor in Space Situational Awareness, Department of Mechanical \& Aerospace Engineering. Email: johnc@buffalo.edu. Fellow AIAA.

${ }^{\dagger}$ Emeritus Engineer, Attitude Control Systems Engineering Branch. Email: Landis.Markley@nasa.gov. Fellow AIAA.
} 


\section{Introduction}

Strapdown gyroscopes have been used for many applications, including spacecraft attitude estimation [1], inertial vehicle navigation [2], underwater vehicle navigation [3], robotic navigation [4], and human navigation systems [5], to name a few. All of these applications require attitude information. Traditional attitude estimation uses a combination of attitude sensor information, such as star trackers, with angular rate sensors, commonly known as gyroscopes [6]. Many spacecraft, including virtually all spacecraft with stringent pointing and/or maneuvering requirements, are provided with accurate gyros, which are the most crucial of all the attitude sensors. A great number of attitude filters incorporate gyro information as part of the dynamic model rather than using the gyro information as a Kalman measurement update. This alternative is often referred to as using gyros in the dynamicmodel replacement mode [7]. The reasons for favoring this method are twofold. Firstly, gyro information may well be much more accurate than the available models of rotational dynamics and torques, and inaccurate dynamic models could actually corrupt the gyro data. The second reason for using gyros in dynamic replacement mode, which is particularly important for onboard filtering, is that it requires much less computation.

The attitude kinematics differential equations are a function of the attitude and angular rate. Therefore, using traditional gyros in dynamic-model replacement mode can be done theoretically by simply replacing the angular rate in the kinematics model with the gyro measurement model. Many types of gyros exist, which can be broadly classified by the physical mechanisms they use: spinning-mass gyros, optical gyros, or Coriolis vibratory gyros. Rate-integrating gyros (RIGs) generally offer a number of unique advantages compared to conventional rate gyroscopes, including mechanically unlimited dynamic range, low noise due to degenerate mode operation, and exceptional scale factor stability [8]. These gyros

do not directly measure angular rate, but rather accumulate angular displacements [9]. The first RIGs were floating type that had a capability of achieving a drift performance of around $0.01 \mathrm{deg} /$ hour [10]. They also exhibit angle output white noise, also known as readout noise or electronic noise, in addition to the usual white noise associated with standard gyros [6].

The most common attitude estimator is based on the Kalman filter. For example, the Kalman filter has been used on numerous spacecraft as the main algorithm to determine attitude and angular rates [1]. Gyros that read angular rates can easily be put into the Kalman filter framework [11]. However, this is not straightforward with RIGs. Readout noise may be large in some RIGs, which causes estimates to degrade. Therefore, this noise must be properly accounted for in the filter design. Reference [12] shows a steady-state Kalman filter formulation using spacecraft attitude sensors coupled with RIGs. The state vector consists of the attitude and RIG biases. However, as shown in this paper, this approach 
ignores correlations which may overestimate the contributions of the RIG output noise to the angle variance.

Reference [13] provides an analytic steady-state solution of the expected performance of the Kalman filter using RIGs for a single-axis case. It is shown in that work that when the readout noise is zero, then the expected performance is identical the single-axis solution shown in Ref. [14] for gyros that read angular rates. The purpose of the present work is to extend Ref. [13] to the three-axis case. This involves an augmentation of the standard gyro model state-vector in order to use the angle outputs of the RIGs in dynamic-model replacement mode. The attitude parameterization here is based on the quaternion [15] in a multiplicative extended Kalman filter framework [7,11]. A reduced-order filter is also derived that ignores the aforementioned correlations. This form is different from the one shown in Ref. [12], which uses the attitude matrix for the attitude parameterization and a steady-state gain. Simulation results are shown to assess the attitude estimation accuracy, and are also compared with the analytic single-axis solutions. Furthermore, the filters are compared to assess the circumstances under which the reduced-order form produces results that are nearly identical to the augmented state-vector form.

The content of this paper is organized as follows. First, the attitude kinematics and sensor models are reviewed. This is followed by a revisit of single-axis analysis that shows the effects of ignoring correlations for the reduced-order state formulation. Then, the equations for the three-axis attitude estimation using RIGs are derived. Both the augmented and reduced-order forms are shown. Finally, simulation results using a star tracker and RIGs for spacecraft attitude estimation are shown.

\section{Attitude Kinematics and Sensor Models}

This section presents a brief review of the attitude kinematics equation of motion using quaternions, and of attitude-vector and RIG sensor models. The quaternion is defined by

$\mathbf{q} \triangleq\left[\begin{array}{ll}\boldsymbol{\varrho}^{T} & q_{4}\end{array}\right]^{T}$, with $\varrho \triangleq\left[\begin{array}{lll}q_{1} & q_{2} & q_{3}\end{array}\right]^{T}=\hat{\mathbf{e}} \sin (\vartheta / 2)$ and $q_{4}=\cos (\vartheta / 2)$, where $\hat{\mathbf{e}}$ is the axis of rotation and $\vartheta$ is the angle of rotation [15]. Since a four-dimensional vector is used to describe three rotational degrees of freedom, the quaternion components cannot be independent of each other. The quaternion satisfies a single constraint given by $\mathbf{q}^{T} \mathbf{q}=1$. The attitude matrix is related to the quaternion by

$$
A(\mathbf{q})=\Xi^{T}(\mathbf{q}) \Psi(\mathbf{q})
$$


with

$$
\begin{aligned}
& \Xi(\mathbf{q}) \triangleq\left[\begin{array}{c}
q_{4} I_{3}+[\boldsymbol{\varrho} \times] \\
-\boldsymbol{\varrho}^{T}
\end{array}\right] \\
& \Psi(\mathbf{q}) \triangleq\left[\begin{array}{c}
q_{4} I_{3}-[\boldsymbol{\varrho} \times] \\
-\boldsymbol{\varrho}^{T}
\end{array}\right]
\end{aligned}
$$

where $I_{3}$ is a $3 \times 3$ identity matrix, and $[\boldsymbol{\varrho} \times]$, called the cross product matrix because $\mathbf{a} \times \mathbf{b}=[\mathbf{a} \times] \mathbf{b}$, is defined as

$$
[\mathbf{a} \times] \triangleq\left[\begin{array}{ccc}
0 & -a_{3} & a_{2} \\
a_{3} & 0 & -a_{1} \\
-a_{2} & a_{1} & 0
\end{array}\right]
$$

Successive rotations can be accomplished using quaternion multiplication. Here the convention of Refs. [11] and [15] is adopted where the quaternions are multiplied in the same order as the attitude matrix multiplication: $A\left(\mathbf{q}^{\prime}\right) A(\mathbf{q})=A\left(\mathbf{q}^{\prime} \otimes \mathbf{q}\right)$. The composition of the quaternions is bilinear, with

$$
\mathbf{q}^{\prime} \otimes \mathbf{q}=\left[\Psi\left(\mathbf{q}^{\prime}\right) \quad \vdots \quad \mathbf{q}^{\prime}\right] \mathbf{q}=[\Xi(\mathbf{q}) \quad \vdots \quad \mathbf{q}] \mathbf{q}^{\prime}
$$

The inverse quaternion is given by $\mathbf{q}^{-1}=\left[\begin{array}{ll}-\varrho^{T} & q_{4}\end{array}\right]^{T}$, and the quaternion kinematics equation is given by

$$
\dot{\mathbf{q}}=\frac{1}{2}\left[\begin{array}{l}
\boldsymbol{\omega} \\
0
\end{array}\right] \otimes \mathbf{q} \triangleq \frac{1}{2}[\boldsymbol{\omega} \otimes] \mathbf{q}
$$

where $\boldsymbol{\omega}$ is the $3 \times 1$ angular rate vector.

Discrete-time unit-vector attitude observations for a single sensor are given by

$$
\tilde{\mathbf{b}}_{i}=A(\mathbf{q}) \mathbf{r}_{i}+\boldsymbol{v}_{i}
$$

where $\tilde{\mathbf{b}}_{i}$ denotes the $i^{\text {th }} 3 \times 1$ measurement vector in the body frame, and $\mathbf{r}_{i}$ is the $i^{\text {th }}$ known $3 \times 1$ reference vector. The sensor error-vector $\boldsymbol{v}_{i}$ is assumed to be zero-mean and approximately Gaussian, satisfying

$$
\begin{gathered}
E\left\{\boldsymbol{v}_{i}\right\}=\mathbf{0}_{3} \\
R_{i} \triangleq E\left\{\boldsymbol{v}_{i} \boldsymbol{v}_{i}^{T}\right\}=\sigma^{2}\left[I_{3}-\left(A \mathbf{r}_{i}\right)\left(A \mathbf{r}_{i}\right)^{T}\right]
\end{gathered}
$$


where $E\{\}$ denotes expectation, and where $\mathbf{0}_{3}$ denotes a $3 \times 1$ vector of zeros. The measurement model expressed by Eq. (7b), known as the QUEST measurement model [16,17], is quite accurate for small field-of-view sensors. Its approximations are discussed in Refs. [18] and [19], and it has been expanded for large fields-of-view in Ref. [19]. Equation (7b) gives a rank-deficient $R$ matrix, which would appear to give rise to problems in an estimator such as the extended Kalman filter (EKF), [20] so this paper uses the simpler, full-rank form

$$
R=\sigma^{2} I_{3}
$$

which has been shown to give equivalent results in this context $[7,16,21]$. A set of $N$ vector measurements can be concatenated to form the $(3 N \times 1)$-component vector

$$
\begin{gathered}
\tilde{\mathbf{y}}_{k}=\left.\left[\begin{array}{c}
A(\mathbf{q}) \mathbf{r}_{1} \\
A(\mathbf{q}) \mathbf{r}_{2} \\
\vdots \\
A(\mathbf{q}) \mathbf{r}_{N}
\end{array}\right]\right|_{t_{k}}+\left.\left[\begin{array}{c}
\boldsymbol{v}_{1} \\
\boldsymbol{v}_{2} \\
\vdots \\
\boldsymbol{v}_{N}
\end{array}\right]\right|_{t_{k}} \\
R_{k}=\operatorname{blkdiag}\left[\begin{array}{llll}
\sigma_{1}^{2} I_{3} & \sigma_{2}^{2} I_{3} & \ldots & \sigma_{N}^{2} I_{3}
\end{array}\right]
\end{gathered}
$$

where blkdiag denotes a block diagonal matrix.

The vehicle is assumed to be equipped with $n$ RIGs, which accumulate an $n$-component vector $\boldsymbol{\varphi}$ of angles modeled by [13]

$$
\begin{gathered}
\dot{\boldsymbol{\varphi}}=M \boldsymbol{\omega}+\boldsymbol{\beta}+\boldsymbol{\eta}_{v} \\
\dot{\boldsymbol{\beta}}=\boldsymbol{\eta}_{u}
\end{gathered}
$$

where $\boldsymbol{\beta}$ is an $n$-component vector of biases, and $M$ is an $n \times 3$ matrix containing nominal gyro alignments, gyro misalignments, and scale factors. It is a general matrix, with the proviso that it must have rank three. In the simplest case, there are three gyros and $M$ is the $3 \times 3$ identity matrix. The $n$-component vectors $\boldsymbol{\eta}_{u}$ and $\boldsymbol{\eta}_{v}$ represent uncorrelated Gaussian white-noise processes satisfying

$$
\begin{aligned}
& E\left\{\boldsymbol{\eta}_{u}(t) \boldsymbol{\eta}_{u}^{T}(\tau)\right\}=Q_{u} \delta(t-\tau) \\
& E\left\{\boldsymbol{\eta}_{v}(t) \boldsymbol{\eta}_{v}^{T}(\tau)\right\}=Q_{v} \delta(t-\tau)
\end{aligned}
$$

where $\delta(t-\tau)$ denotes the Dirac delta function, and where $Q_{u}$ and $Q_{v}$ are diagonal $n \times n$ 
spectral density matrices. Measurements of $\varphi$ are given by

$$
\tilde{\varphi}=\varphi+\mathbf{v}_{e}
$$

where $\mathbf{v}_{e}$ is a vector of $n$ uncorrelated Gaussian gyro output measurement errors with diagonal covariance $Q_{e}$.

\section{Single-Axis Analysis}

In the standard attitude estimation EKF [11] the state vector consists of the attitude and gyro biases. In this section, the single-axis analysis is revisited to show the reason why an augmented state vector should be employed when using RIGs in the EKF setting. The true single-axis attitude angle $\vartheta$ obeys the kinematic equation

$$
\dot{\vartheta}=\omega
$$

where $\omega$ is the true single-axis angular rate. The single-axis RIG model is given by

$$
\begin{gathered}
\dot{\varphi}=\omega+\beta+\eta_{v} \\
\dot{\beta}=\eta_{u}
\end{gathered}
$$

where the spectral densities of $\eta_{v}$ and $\eta_{u}$ are given by $\sigma_{v}^{2}$ and $\sigma_{u}^{2}$, respectively. The state vector is given by $\mathbf{x}=\left[\begin{array}{lll}\vartheta & \beta & \varphi\end{array}\right]^{T}$, and the corresponding estimate is given by $\hat{\mathbf{x}}=\left[\begin{array}{lll}\hat{\vartheta} & \hat{\beta} & \hat{\varphi}\end{array}\right]^{T}$. Thus the three-component state $\mathbf{x}$ obeys the discrete-time propagation equation

$$
\mathbf{x}_{k+1}=\left[\begin{array}{ccc}
1 & 0 & 0 \\
0 & 1 & 0 \\
0 & \delta t & 1
\end{array}\right] \mathbf{x}_{k}+\left[\begin{array}{l}
1 \\
0 \\
1
\end{array}\right] \int_{t_{k}}^{t_{k+1}} \omega(\tau) d \tau+\left[\begin{array}{c}
0 \\
N_{u}\left(t_{k+1}, t_{k}\right) \\
N_{v}\left(t_{k+1}, t_{k}\right)
\end{array}\right]
$$

where $\delta t \triangleq t_{k+1}-t_{k}$ is not assumed to be infinitesimal, and the quantities $N_{u}$ and $N_{v}$ are defined by

$$
\begin{aligned}
& N_{u}\left(t_{k+1}, t_{k}\right)=\int_{t_{k}}^{t_{k+1}} \eta_{u}(\tau) d \tau \\
& N_{v}\left(t_{k+1}, t_{k}\right)=\int_{t_{k}}^{t_{k+1}}\left[\eta_{v}(\tau)+\left(t_{k+1}-\tau\right) \eta_{u}(\tau)\right] d \tau
\end{aligned}
$$

Some computations from the Appendix have been used in deriving Eqs. (16). 
The last line of Eq. (15) shows that the unknown quantity $\int_{t_{k}}^{t_{k+1}} \omega(\tau) d \tau$ is given by

$$
\int_{t_{k}}^{t_{k+1}} \omega(\tau) d \tau=\varphi_{k+1}-\left[\begin{array}{lll}
0 & \delta t & 1
\end{array}\right] \mathbf{x}_{k}-N_{v}\left(t_{k+1}, t_{k}\right)
$$

The RIG measurement at the end of the propagation interval is modeled as

$$
\tilde{\varphi}_{k+1}=\varphi_{k+1}+v_{e}
$$

where $v_{e}$ is a zero-mean Gaussian measurement noise with variance $\sigma_{e}^{2}$. It is assumed that $\eta_{u}, \eta_{v}$, and $v_{e}$ are uncorrelated. Substituting Eqs. (17) and (18) into Eq. (15) to eliminate unknown quantities gives [7]

$$
\mathbf{x}_{k+1}=\Phi(\delta t) \mathbf{x}_{k}+\left[\begin{array}{l}
1 \\
0 \\
1
\end{array}\right] \tilde{\varphi}_{k+1}+\left[\begin{array}{c}
-N_{v}\left(t_{k+1}, t_{k}\right)-v_{e} \\
N_{u}\left(t_{k+1}, t_{k}\right) \\
-v_{e}
\end{array}\right]
$$

where

$$
\Phi(\delta t)=\left[\begin{array}{ccc}
1 & -\delta t & -1 \\
0 & 1 & 0 \\
0 & 0 & 0
\end{array}\right]
$$

The state estimate obeys

$$
\hat{\mathbf{x}}_{k+1}=\Phi(\delta t) \hat{\mathbf{x}}_{k}+\left[\begin{array}{l}
1 \\
0 \\
1
\end{array}\right] \tilde{\varphi}_{k+1}
$$

Defining the state error vector $\Delta \mathbf{x} \triangleq \mathbf{x}-\hat{\mathbf{x}}$ leads to

$$
\Delta \mathbf{x}_{k+1}=\Phi(\delta t) \Delta \mathbf{x}_{k}+\left[\begin{array}{c}
-N_{v}\left(t_{k+1}, t_{k}\right)-v_{e} \\
N_{u}\left(t_{k+1}, t_{k}\right) \\
-v_{e}
\end{array}\right]
$$

The error-covariance $P \triangleq E\left\{\Delta \mathbf{x} \Delta \mathbf{x}^{T}\right\}$ propagates according to

$$
P_{k+1}=\Phi(\delta t) P_{k} \Phi^{T}(\delta t)+Q(\delta t)
$$


where the process noise covariance $Q$ is given by

$$
\begin{aligned}
Q(\delta t) & =E\left\{\left[\begin{array}{c}
-N_{v}\left(t_{k+1}, t_{k}\right)-v_{e} \\
N_{u}\left(t_{k+1}, t_{k}\right) \\
-v_{e}
\end{array}\right]\left[\begin{array}{c}
-N_{v}\left(t_{k+1}, t_{k}\right)-v_{e} \\
N_{u}\left(t_{k+1}, t_{k}\right) \\
-v_{e}
\end{array}\right]^{T}\right\} \\
& =\left[\begin{array}{ccc}
\sigma_{v}^{2} \delta t+\frac{1}{3} \sigma_{u}^{2} \delta t^{3}+\sigma_{e}^{2} & -\frac{1}{2} \sigma_{u}^{2} \delta t^{2} & \sigma_{e}^{2} \\
-\frac{1}{2} \sigma_{u}^{2} \delta t^{2} & \sigma_{u}^{2} \delta t & 0 \\
\sigma_{e}^{2} & 0 & \sigma_{e}^{2}
\end{array}\right]
\end{aligned}
$$

The superscripts - and +, which are generally used to distinguish pre-update and postupdate quantities, do not appear in these equations because the dynamic model replacement mode effectively combines a dynamic propagation and a gyro measurement update in a single step. These superscripts will make their appearance when a discrete-time attitude measurement update is considered.

It is easy to show by mathematical induction that propagation by $\ell$ steps gives

$$
P_{k+\ell}=\Phi(\ell \delta t) P_{k} \Phi^{T}(\ell \delta t)+Q(\ell \delta t)
$$

This equation has two interesting properties. The first is that it depends only on the total propagation time $\ell \delta t$, not on $\ell$ and $\delta t$ separately. The second is that the noise term $\sigma_{e}$ does not accumulate, so that the covariance only depends on the output noise of the last readout.

Equation (19) obtains $\hat{\varphi}_{k}$ from the state vector $\mathbf{x}_{k}$. Another approach is to assume that $\hat{\varphi}_{k}=\tilde{\varphi}_{k}$, and $\Delta \varphi_{k}=v_{e_{k}}$, where $v_{e_{k}}$ is zero-mean Gaussian measurement noise with variance $\sigma_{e}^{2}$ independent of $v_{e_{k+1}}$, which has simply been called $v_{e}$ up to this point. With these modifications, $\varphi_{k}$ can be omitted from the state vector, leaving a two-component (reduced) state vector $\mathbf{x}^{r}=[\vartheta \quad \beta]^{T}$, and

$$
\begin{gathered}
\hat{\mathbf{x}}_{k+1}^{r}=\tilde{\Phi}(\delta t) \hat{\mathbf{x}}_{k}^{r}+\left[\begin{array}{l}
1 \\
0
\end{array}\right]\left(\tilde{\varphi}_{k+1}-\tilde{\varphi}_{k}\right) \\
\Delta \mathbf{x}_{k+1}^{r}=\tilde{\Phi}(\delta t) \Delta \mathbf{x}_{k}^{r}+\left[\begin{array}{c}
-N_{v}\left(t_{k+1}, t_{k}\right)-v_{e_{k+1}}+v_{e_{k}} \\
N_{u}\left(t_{k+1}, t_{k}\right)
\end{array}\right]
\end{gathered}
$$

where

$$
\tilde{\Phi}(\delta t)=\left[\begin{array}{cc}
1 & -\delta t \\
0 & 1
\end{array}\right]
$$

This would seem to be an improvement, because it reduces the size of the state vector and 
covariance matrix. It is somewhat ad hoc, though, because it is not completely clear what to use as a replacement for Eq. (19). Another and more significant problem is that the measurement noises at times $t_{k}$ and $t_{k+1}$ are correlated.

The reduced error-covariance follows

$$
P_{k+1}^{r}=\tilde{\Phi}(\delta t) P_{k}^{r} \tilde{\Phi}^{T}(\delta t)+Q^{r}(\delta t)
$$

where

$$
\begin{aligned}
Q^{r}(\delta t) & =E\left\{\left[\begin{array}{c}
-N_{v}-v_{e_{k+1}}+v_{e_{k}} \\
N_{u}
\end{array}\right]\left[\begin{array}{r}
-N_{v}-v_{e_{k+1}}+v_{e_{k}} \\
N_{u}
\end{array}\right]^{T}\right\} \\
& =\left[\begin{array}{cc}
\sigma_{v}^{2} \delta t+\frac{1}{3} \sigma_{u}^{2} \delta t^{3}+2 \sigma_{e}^{2} & -\frac{1}{2} \sigma_{u}^{2} \delta t^{2} \\
-\frac{1}{2} \sigma_{u}^{2} \delta t^{2} & \sigma_{u}^{2} \delta t
\end{array}\right]
\end{aligned}
$$

Iterating this equation $\ell$ times gives

$$
\begin{aligned}
P_{k+\ell}^{r} & =\tilde{\Phi}(\ell \delta t) P_{k}^{r} \tilde{\Phi}^{T}(\ell \delta t) \\
& +\left[\begin{array}{cc}
\sigma_{v}^{2}(\ell \delta t)+\frac{1}{3} \sigma_{u}^{2}(\ell \delta t)^{3}+2 \ell \sigma_{e}^{2} & -\frac{1}{2} \sigma_{u}^{2}(\ell \delta t)^{2} \\
-\frac{1}{2} \sigma_{u}^{2}(\ell \delta t)^{2} & \sigma_{u}^{2}(\ell \delta t)
\end{array}\right]
\end{aligned}
$$

This shows that ignoring the correlations in the measurements greatly overestimates the contributions of the RIG output noise to the angle variance. The upper left corner of $Q(\ell \delta t)$ in Eq. (25) contains the contribution $\sigma_{e}^{2}$, while the upper left corner of the corresponding matrix in Eq. (30) contains the contribution $2 \ell \sigma_{e}^{2}$. Correct handling of the correlations causes the successive measurement output errors to cancel out, leaving only the last one. This provides the motivation for augmenting the state vector employed in the standard attitude estimation EKF of Ref. [11], which assumes direct measurements of the angular rate instead of the RIG-type measurements that are assumed here.

\section{Rate-Integrating Gyro-Based Kalman Filter}

In this section, the RIG-based multiplicative extended Kalman filter (MEKF) is derived. First, the propagation equations are derived, and then the update equations are shown. 


\section{Propagation Equations}

The $4+2 n$-component "global" truth state vector is given by

$$
\mathrm{x}^{\mathrm{big}}=\left[\begin{array}{l}
\mathrm{q} \\
\beta \\
\varphi
\end{array}\right]
$$

where $\boldsymbol{\beta}$ is an $n$-component vector of gyro drift biases, and $\boldsymbol{\varphi}$ is the $n$-component vector of angles accumulated internally by the RIGs. The components of the global state vector obey the following truth-dynamics equations:

$$
\begin{gathered}
\dot{\mathbf{q}}=\frac{1}{2}[\boldsymbol{\omega} \otimes] \mathbf{q} \\
\dot{\boldsymbol{\beta}}=\boldsymbol{\eta}_{u} \\
\dot{\boldsymbol{\varphi}}=M \boldsymbol{\omega}+\boldsymbol{\beta}+\boldsymbol{\eta}_{v}
\end{gathered}
$$

where $\boldsymbol{\omega}$ is the true angular rate vector. Equation (32) is the generalization of Eqs. (13) and (14) to three space dimensions and $n$ gyros. Note that the angular rate vector does not appear as a component of the state vector. The global state estimates obey the following dynamic equations:

$$
\begin{gathered}
\dot{\hat{\mathbf{q}}}=\frac{1}{2}[\hat{\boldsymbol{\omega}} \otimes] \hat{\mathbf{q}} \\
\dot{\hat{\boldsymbol{\beta}}}=\mathbf{0}_{3} \\
\dot{\hat{\boldsymbol{\varphi}}}=M \hat{\boldsymbol{\omega}}+\hat{\boldsymbol{\beta}}
\end{gathered}
$$

The finite-time propagation of these equations is

$$
\begin{gathered}
\hat{\mathbf{q}}_{k+1}=\exp \left(\frac{1}{2}\left[\int_{t_{k}}^{t_{k+1}} \hat{\boldsymbol{\omega}}(\tau) d \tau \otimes\right]\right) \hat{\mathbf{q}}_{k} \\
\hat{\boldsymbol{\beta}}_{k+1}=\hat{\boldsymbol{\beta}}_{k} \\
\hat{\boldsymbol{\varphi}}_{k+1}=\hat{\boldsymbol{\varphi}}_{k}+M \int_{t_{k}}^{t_{k+1}} \hat{\boldsymbol{\omega}}(\tau) d \tau+\hat{\boldsymbol{\beta}} \delta t
\end{gathered}
$$

Equation (34a) requires the customary assumption that any change in the orientation of the rotation axis over the time interval $\delta t=t_{k+1}-t_{k}$ is negligible. The quantity $\hat{\boldsymbol{\beta}}$ is written without a time argument in Eq. (34c) and all the subsequent equations, because Eq. (33b) shows that it is constant between gyro measurements.

The essence of using RIGs in dynamic-model replacement mode is to obtain the angular 
rates from the gyros. Thus the integrated rates are regarded as the unknowns rather than $\hat{\boldsymbol{\varphi}}_{k+1}$ in Eq. (34c), and this equation is solved for these quantities to obtain

$$
\hat{\boldsymbol{\psi}}_{k+1, k} \triangleq \int_{t_{k}}^{t_{k+1}} \hat{\boldsymbol{\omega}}(\tau) d \tau=M^{L}\left(\tilde{\boldsymbol{\varphi}}_{k+1}-\hat{\boldsymbol{\varphi}}_{k}-\hat{\boldsymbol{\beta}} \delta t\right)
$$

where $M^{L}$ is a left inverse of $M$, i.e. a matrix satisfying $M^{L} M=I_{3}$. This matrix will be discussed in detail later.

Substituting Eq. (35) into Eq. (34a) leads to [7]

$$
\begin{aligned}
\hat{\mathbf{q}}_{k+1} & =\exp \left(\frac{1}{2}\left[\hat{\boldsymbol{\psi}}_{k+1, k} \otimes\right]\right) \hat{\mathbf{q}}_{k} \\
& =\left\{\cos \left(\frac{\hat{\psi}_{k+1, k}}{2}\right) I_{4}+\sin \left(\frac{\hat{\psi}_{k+1, k}}{2}\right)\left[\hat{\mathbf{e}}_{k+1, k} \otimes\right]\right\} \hat{\mathbf{q}}_{k}
\end{aligned}
$$

where $I_{4}$ is a $4 \times 4$ identity matrix, and the rotation angle $\hat{\psi}_{k+1, k}$ and rotation axis unit vector $\hat{\mathbf{e}}_{k+1, k}$ have the explicit forms

$$
\begin{gathered}
\hat{\psi}_{k+1, k}=\left\|M^{L} \hat{\boldsymbol{\varphi}}_{k+1}-M^{L} \hat{\boldsymbol{\varphi}}_{k}-M^{L} \hat{\boldsymbol{\beta}} \delta t\right\| \\
\hat{\mathbf{e}}_{k+1, k}=\left[M^{L} \hat{\boldsymbol{\varphi}}_{k+1}-M^{L} \hat{\boldsymbol{\varphi}}_{k}-M^{L} \hat{\boldsymbol{\beta}} \delta t\right] / \hat{\psi}_{k+1, k}
\end{gathered}
$$

These forms are chosen to show that the attitude estimator does not need to know the $n$ component vectors $\hat{\boldsymbol{\beta}}$ and $\hat{\boldsymbol{\varphi}}$, but only the three-component vectors $M^{L} \hat{\boldsymbol{\beta}}$ and $M^{L} \hat{\boldsymbol{\varphi}}$. Thus the effective state is the ten-component vector

$$
\mathbf{x}=\left[\begin{array}{c}
\mathbf{q} \\
M^{L} \boldsymbol{\beta} \\
M^{L} \boldsymbol{\varphi}
\end{array}\right]
$$

Equations (36) and (37) show that the quaternion propagation needs the quantities $M^{L} \hat{\boldsymbol{\beta}}$, $M^{L} \hat{\boldsymbol{\varphi}}_{k}$, and $M^{L} \hat{\boldsymbol{\varphi}}_{k+1}$. The values of $M^{L} \hat{\boldsymbol{\beta}}$ and $M^{L} \hat{\boldsymbol{\varphi}}_{k}$ are retained from the previous update, which may be from a gyro measurement or a measurement by some different sensor. The dynamic-model replacement mode for RIGs sets $M^{L} \hat{\boldsymbol{\varphi}}_{k+1}=M^{L} \tilde{\boldsymbol{\varphi}}_{k+1}$, where $\tilde{\boldsymbol{\varphi}}_{k+1}$ is the vector of RIG outputs at time $t_{k+1}$. This substitution makes it unnecessary to propagate the estimates of the RIG accumulated angles, and it has the result that the propagation of the state estimates is straightforward with the usual fixed-axis approximation for closed-form quaternion propagation.

This is analogous to using the vector of rate gyro outputs, denoted by $\tilde{\boldsymbol{\omega}}(t)$, to compute the rate estimate $\hat{\boldsymbol{\omega}}(t)=M^{L}[\tilde{\boldsymbol{\omega}}(t)-\hat{\boldsymbol{\beta}}]$ when rate gyros are used in dynamic-model replace- 
ment mode [11]. In this application, the integral in Eq. (34a) is evaluated continuously in principle, but uses some kind of low-order hold of discretely sampled rate gyro outputs in practice. The RIG propagation does not require any kind of hold, because the RIGs actually perform the continuous integration of the components along their input axes of the true body rates over the time interval $\delta t$.

The MEKF represents the attitude error in terms of a three-vector $\boldsymbol{\delta} \boldsymbol{\vartheta}$ as $[7,11]$

$$
\mathbf{q}=\boldsymbol{\delta} \mathbf{q}(\boldsymbol{\delta} \boldsymbol{\vartheta}) \otimes \hat{\mathbf{q}}
$$

so that a reduced, nine-component, "local" error-state vector can be used, which is given by

$$
\Delta \mathrm{x}=\left[\begin{array}{c}
\boldsymbol{\delta} \vartheta \\
M^{L} \Delta \boldsymbol{\beta} \\
M^{L} \Delta \varphi
\end{array}\right]
$$

where $\Delta \boldsymbol{\beta} \triangleq \boldsymbol{\beta}-\hat{\boldsymbol{\beta}}$ and $\Delta \boldsymbol{\varphi} \triangleq \boldsymbol{\varphi}-\hat{\boldsymbol{\varphi}}$. Note that the MEKF defines $\boldsymbol{\delta} \boldsymbol{\vartheta}$ by Eq. (39), not as the difference between a true value and an expectation. The components of $\Delta \mathbf{x}$ obey the dynamic equations $[7,11]$

$$
\begin{gathered}
\boldsymbol{\delta} \dot{\boldsymbol{\vartheta}}=-[\hat{\boldsymbol{\omega}} \times] \boldsymbol{\delta} \boldsymbol{\vartheta}+\Delta \boldsymbol{\omega} \\
M^{L} \Delta \dot{\boldsymbol{\beta}}=M^{L} \boldsymbol{\eta}_{u} \\
M^{L} \Delta \dot{\boldsymbol{\varphi}}=M^{L}\left(M \Delta \boldsymbol{\omega}+\Delta \boldsymbol{\beta}+\boldsymbol{\eta}_{v}\right)=\Delta \boldsymbol{\omega}+M^{L} \Delta \boldsymbol{\beta}+M^{L} \boldsymbol{\eta}_{v}
\end{gathered}
$$

where $\Delta \boldsymbol{\omega} \triangleq \omega-\hat{\boldsymbol{\omega}}$.

Equation (41) can be written in matrix form as

$$
\Delta \dot{\mathbf{x}}=F \Delta \mathbf{x}+\left[\begin{array}{c}
I_{3} \\
0_{3 \times 3} \\
I_{3}
\end{array}\right] \Delta \boldsymbol{\omega}+\left[\begin{array}{c}
\mathbf{0}_{3} \\
M^{L} \boldsymbol{\eta}_{u} \\
M^{L} \boldsymbol{\eta}_{v}
\end{array}\right]
$$

where $0_{3 \times 3}$ is a $3 \times 3$ matrix of zeros, and

$$
F \triangleq\left[\begin{array}{ccc}
-[\hat{\boldsymbol{\omega}} \times] & 0_{3 \times 3} & 0_{3 \times 3} \\
0_{3 \times 3} & 0_{3 \times 3} & 0_{3 \times 3} \\
0_{3 \times 3} & I_{3} & 0_{3 \times 3}
\end{array}\right]
$$


If $\Phi\left(t, t_{0}\right)$ is the solution of $\dot{\Phi}\left(t, t_{0}\right)=F \Phi\left(t, t_{0}\right)$ with initial condition $\Phi\left(t_{0}, t_{0}\right)=I_{9}$, then

$$
\Delta \mathbf{x}_{k+1}=\Phi\left(t_{k+1}, t_{k}\right) \Delta \mathbf{x}_{k}+\int_{t_{k}}^{t_{k+1}} \Phi\left(t_{k+1}, \tau\right)\left(\left[\begin{array}{c}
I_{3} \\
0_{3 \times 3} \\
I_{3}
\end{array}\right] \Delta \boldsymbol{\omega}(\tau)+\left[\begin{array}{c}
\mathbf{0}_{3} \\
M^{L} \boldsymbol{\eta}_{u}(\tau) \\
M^{L} \boldsymbol{\eta}_{v}(\tau)
\end{array}\right]\right) d \tau
$$

The state transition matrix $\Phi\left(t_{k+1}, t_{k}\right)$ is given by

$$
\Phi\left(t_{k+1}, t_{k}\right)=\left[\begin{array}{ccc}
\Phi_{\vartheta \vartheta}\left(t_{k+1}, t_{k}\right) & 0_{3 \times 3} & 0_{3 \times 3} \\
0_{3 \times 3} & I_{3} & 0_{3 \times 3} \\
0_{3 \times 3} & \delta t I_{3} & I_{3}
\end{array}\right]
$$

where $\dot{\Phi}_{\vartheta \vartheta}\left(t, t_{0}\right)=-[\hat{\boldsymbol{\omega}} \times] \Phi_{\vartheta \vartheta}\left(t, t_{0}\right)$ with initial condition $\Phi_{\vartheta \vartheta}\left(t_{0}, t_{0}\right)=I_{3}$. Assuming again that any motion of the rotation axis over $\delta t$ is negligible gives [7]

$$
\Phi_{\vartheta \vartheta}\left(t_{k+1}, t_{k}\right)=I_{3}-\sin \left(\hat{\psi}_{k+1, k}\right)\left[\hat{\mathbf{e}}_{k+1, k} \times\right]+\left[1-\cos \left(\hat{\psi}_{k+1, k}\right)\right]\left[\hat{\mathbf{e}}_{k+1, k} \times\right]^{2}
$$

with $\hat{\psi}_{k+1, k}$ and $\hat{\mathbf{e}}_{k+1, k}$ given by Eq. (37). Substituting Eq. (45) into Eq. (44) yields

$$
\Delta \mathbf{x}_{k+1}=\Phi\left(t_{k+1}, t_{k}\right) \Delta \mathbf{x}_{k}+\int_{t_{k}}^{t_{k+1}}\left[\begin{array}{c}
\Phi_{\vartheta \vartheta}\left(t_{k+1}, \tau\right) \\
0_{3 \times 3} \\
I_{3}
\end{array}\right] \Delta \boldsymbol{\omega}(\tau) d \tau+\left[\begin{array}{c}
\mathbf{0}_{3} \\
M^{L} \mathbf{N}_{u}\left(t_{k+1}, t_{k}\right) \\
M^{L} \mathbf{N}_{v}\left(t_{k+1}, t_{k}\right)
\end{array}\right]
$$

where

$$
\begin{aligned}
& \mathbf{N}_{u}\left(t_{k+1}, t_{k}\right)=\int_{t_{k}}^{t_{k+1}} \boldsymbol{\eta}_{u}(\tau) d \tau \\
& \mathbf{N}_{v}\left(t_{k+1}, t_{k}\right)=\int_{t_{k}}^{t_{k+1}}\left[\boldsymbol{\eta}_{v}(\tau)+\left(t_{k+1}-\tau\right) \boldsymbol{\eta}_{u}(\tau)\right] d \tau
\end{aligned}
$$

The bottom three rows of Eq. (47) give

$$
\int_{t_{k}}^{t_{k+1}} \Delta \boldsymbol{\omega}(\tau) d \tau=\left[\begin{array}{lll}
0_{3 \times 3} & 0_{3 \times 3} & I_{3}
\end{array}\right] \Delta \mathbf{x}_{k+1}-\left[\begin{array}{lll}
0_{3 \times 3} & \delta t I_{3} & I_{3}
\end{array}\right] \Delta \mathbf{x}_{k}-M^{L} \mathbf{N}_{v}\left(t_{k+1}, t_{k}\right)
$$

This equation follows directly from Eqs. (33c), (34c), and (35), and the definitions of $\Delta \boldsymbol{\beta}, \Delta \boldsymbol{\varphi}, \Delta \boldsymbol{\omega}$, and $\mathbf{N}_{v}$. It is not possible to simply substitute this into Eq. (47) as was done in deriving Eq. (19), because the first three rows of Eq. (47) contain the integral 
$\int_{t_{k}}^{t_{k+1}} \Phi_{\vartheta \vartheta}\left(t_{k+1}, \tau\right) \Delta \boldsymbol{\omega}(\tau) d \tau$. Accomplishing this substitution requires the approximation:

$$
\int_{t_{k}}^{t_{k+1}} \Phi_{\vartheta \vartheta}\left(t_{k+1}, \tau\right) \Delta \boldsymbol{\omega}(\tau) d \tau \approx \bar{\Phi}\left(t_{k+1}, t_{k}\right) \int_{t_{k}}^{t_{k+1}} \Delta \boldsymbol{\omega}(\tau) d \tau
$$

where $\bar{\Phi}\left(t_{k+1}, t_{k}\right)$ represents some kind of average of $\Phi_{\vartheta \vartheta}\left(t_{k+1}, \tau\right)$ over the integration span. The following form is chosen for $\bar{\Phi}\left(t_{k+1}, t_{k}\right)$ :

$$
\bar{\Phi}\left(t_{k+1}, t_{k}\right)=\frac{1}{\delta t} \int_{t_{k}}^{t_{k+1}} \Phi_{\vartheta \vartheta}\left(t_{k+1}, \tau\right) d \tau
$$

This form has two advantages. The first is that it provides an exact representation of $\int_{t_{k}}^{t_{k+1}} \Phi_{\vartheta \vartheta}\left(t_{k+1}, \tau\right) \Delta \boldsymbol{\omega}(\tau) d \tau$ if $\Delta \boldsymbol{\omega}$ is constant over the integration span. It is not expected that $\Delta \boldsymbol{\omega}$ is constant over the integration span, and the fact that this quantity is unknown is what requires the use of Eq. (49), but the choice for $\bar{\Phi}\left(t_{k+1}, t_{k}\right)$ at least assures that any constant part of $\Delta \boldsymbol{\omega}$ is correctly accounted for. The second advantage of this choice for $\bar{\Phi}\left(t_{k+1}, t_{k}\right)$ is that it leads to an estimator that most closely resembles the conventional MEKF with rate gyros in model replacement mode, as will be seen later. The approximation that $\hat{\boldsymbol{\omega}}$ is constant in both magnitude and direction over the integration span allows the integral to be evaluated in closed-form to obtain

$$
\bar{\Phi}\left(t_{k+1}, t_{k}\right)=I_{3}-\frac{1-\cos \left(\hat{\psi}_{k+1, k}\right)}{\hat{\psi}_{k+1, k}}\left[\hat{\mathbf{e}}_{k+1, k} \times\right]+\frac{\hat{\psi}_{k+1, k}-\sin \left(\hat{\psi}_{k+1, k}\right)}{\hat{\psi}_{k+1, k}}\left[\hat{\mathbf{e}}_{k+1, k} \times\right]^{2}
$$

Some special care must be taken to avoid division by zero if the rotation angle is zero.

Although approximations of the kind used here for the quaternion and covariance propagation are often made simply for computational convenience, something like Eq. (50) is absolutely necessary in this RIG formulation, because the RIGs do not know that they are in a rotating frame; they just integrate the components of the angular rates on their input axes without knowing anything about the rates on the cross axes or Eulerian kinematics. 
Using the approximation of Eq. (50) and then substituting Eq. (49) into Eq. (47) give

$$
\begin{aligned}
\Delta \mathbf{x}_{k+1} & \approx \Phi\left(t_{k+1}, t_{k}\right) \Delta \mathbf{x}_{k}+\left[\begin{array}{c}
\bar{\Phi}\left(t_{k+1}, t_{k}\right) \\
0_{3 \times 3} \\
I_{3}
\end{array}\right] \int_{t_{k}}^{t_{k+1}} \Delta \boldsymbol{\omega}(\tau) d \tau+\left[\begin{array}{c}
\mathbf{0}_{3} \\
M^{L} \mathbf{N}_{u}\left(t_{k+1}, t_{k}\right) \\
M_{L} \mathbf{N}_{v}\left(t_{k+1}, t_{k}\right)
\end{array}\right] \\
& =\Phi\left(t_{k+1}, t_{k}\right) \Delta \mathbf{x}_{k}+\left[\begin{array}{c}
\bar{\Phi}\left(t_{k+1}, t_{k}\right) \\
0_{3 \times 3} \\
I_{3}
\end{array}\right]\left\{\left[\begin{array}{lll}
0_{3 \times 3} & 0_{3 \times 3} & I_{3}
\end{array}\right] \Delta \mathbf{x}_{k+1}\right. \\
& \left.-\left[\begin{array}{lll}
0_{3 \times 3} & \delta t I_{3} & I_{3}
\end{array}\right] \Delta \mathbf{x}_{k}-M^{L} \mathbf{N}_{v}\left(t_{k+1}, t_{k}\right)\right\}+\left[\begin{array}{c}
\mathbf{0}_{3} \\
M^{L} \mathbf{N}_{u}\left(t_{k+1}, t_{k}\right) \\
M_{L} \mathbf{N}_{v}\left(t_{k+1}, t_{k}\right)
\end{array}\right]
\end{aligned}
$$

which gives

$$
\begin{aligned}
{\left[\begin{array}{ccc}
I_{3} & 0_{3 \times 3} & -\bar{\Phi}\left(t_{k+1}, t_{k}\right) \\
0_{3 \times 3} & I_{3} & 0_{3 \times 3} \\
0_{3 \times 3} & 0_{3 \times 3} & 0_{3 \times 3}
\end{array}\right] \Delta \mathbf{x}_{k+1} } & =\left[\begin{array}{ccc}
\Phi_{\vartheta \vartheta}\left(t_{k+1}, t_{k}\right) & -\bar{\Phi}\left(t_{k+1}, t_{k}\right) \delta t & -\bar{\Phi}\left(t_{k+1}, t_{k}\right) \\
0_{3 \times 3} & I_{3} & 0_{3 \times 3} \\
0_{3 \times 3} & 0_{3 \times 3} & 0_{3 \times 3}
\end{array}\right] \Delta \mathbf{x}_{k} \\
+ & {\left[\begin{array}{c}
-\bar{\Phi}\left(t_{k+1}, t_{k}\right) M^{L} \mathbf{N}_{v}\left(t_{k+1}, t_{k}\right) \\
M^{L} \mathbf{N}_{u}\left(t_{k+1}, t_{k}\right) \\
\mathbf{0}_{3}
\end{array}\right] }
\end{aligned}
$$

The bottom three rows of this equation give $\mathbf{0}_{3}=\mathbf{0}_{3}$, so they contain no information. This is not surprising because they have been used to substitute the integral of the angular rate vector into Eq. (47). The middle three rows give a perfectly reasonable equation for $\Delta \boldsymbol{\beta}_{k+1}$. The top three rows give

$$
\begin{aligned}
\boldsymbol{\delta} \boldsymbol{\vartheta}_{k+1}-\bar{\Phi}\left(t_{k+1}, t_{k}\right) M^{L} \Delta \boldsymbol{\varphi}_{k+1} & =\left[\begin{array}{lll}
\Phi_{\vartheta \vartheta}\left(t_{k+1}, t_{k}\right) & -\bar{\Phi}\left(t_{k+1}, t_{k}\right) \delta t & -\bar{\Phi}\left(t_{k+1}, t_{k}\right)
\end{array}\right] \Delta \mathbf{x}_{k} \\
& -\bar{\Phi}\left(t_{k+1}, t_{k}\right) M^{L} \mathbf{N}_{v}\left(t_{k+1}, t_{k}\right)
\end{aligned}
$$

This has expectation

$$
\boldsymbol{\delta} \hat{\vartheta}_{k+1}=\Phi_{\vartheta \vartheta}\left(t_{k+1}, t_{k}\right) \boldsymbol{\delta} \hat{\vartheta}_{k}
$$

because $\Delta \boldsymbol{\beta}, \Delta \boldsymbol{\varphi}$, and $\mathbf{N}_{v}$ are all defined to have zero mean. The MEKF has reset $\boldsymbol{\delta} \hat{\boldsymbol{\vartheta}}$ to zero after the last measurement update, so Eq. (56) says that it remains zero through all 
the following RIG propagation steps. This obviates the need to propagate this expectation, as is always assumed in the MEKF, and it also means that $\boldsymbol{\delta} \boldsymbol{\vartheta}$ really is an error. Equation (55) provides an equation for $\boldsymbol{\delta} \boldsymbol{\vartheta}_{k+1}-\bar{\Phi}\left(t_{k+1}, t_{k}\right) M^{L} \Delta \boldsymbol{\varphi}_{k+1}$, but not for $\boldsymbol{\delta} \boldsymbol{\vartheta}_{k+1}$ and $\Delta \boldsymbol{\varphi}_{k+1}$ separately. More information is clearly needed, which is obtained by recalling that the dynamic-model replacement mode sets $\hat{\boldsymbol{\varphi}}_{k+1}=\tilde{\boldsymbol{\varphi}}_{k+1}$. It follows from Eq. (12) that

$$
M^{L} \Delta \boldsymbol{\varphi}_{k+1}=M^{L}\left[\boldsymbol{\varphi}_{k+1}-\hat{\boldsymbol{\varphi}}_{k+1}\right]=M^{L}\left[\boldsymbol{\varphi}_{k+1}-\tilde{\boldsymbol{\varphi}}_{k+1}\right]=-M^{L} \mathbf{v}_{e}
$$

This is used to replace the information-free bottom three rows of Eq. (54), and is also substituted into the top three rows, giving

$$
\begin{aligned}
& \Delta \mathbf{x}_{k+1}=\left[\begin{array}{ccc}
\Phi_{\vartheta \vartheta}\left(t_{k+1}, t_{k}\right) & -\bar{\Phi}\left(t_{k+1}, t_{k}\right) \delta t & -\bar{\Phi}\left(t_{k+1}, t_{k}\right) \\
0_{3 \times 3} & I_{3} & 0_{3 \times 3} \\
0_{3 \times 3} & 0_{3 \times 3} & 0_{3 \times 3}
\end{array}\right] \Delta \mathbf{x}_{k} \\
& +\operatorname{blkdiag}\left(\left[\begin{array}{lll}
\bar{\Phi}\left(t_{k+1}, t_{k}\right) M^{L} & M^{L} & M^{L}
\end{array}\right]\right)\left[\begin{array}{c}
-\mathbf{N}_{v}\left(t_{k+1}, t_{k}\right)-\mathbf{v}_{e} \\
\mathbf{N}_{u}\left(t_{k+1}, t_{k}\right) \\
-\mathbf{v}_{e}
\end{array}\right] \\
& \triangleq \Phi_{\mathrm{eff}}\left(t_{k+1}, t_{k}\right) \Delta \mathbf{x}_{k}+G\left(t_{k+1}, t_{k}\right)\left[\begin{array}{c}
-\mathbf{N}_{v}\left(t_{k+1}, t_{k}\right)-\mathbf{v}_{e} \\
\mathbf{N}_{u}\left(t_{k+1}, t_{k}\right) \\
-\mathbf{v}_{e}
\end{array}\right]
\end{aligned}
$$

which is the three-axis equivalent of Eq. (22).

Note that the quantity $\mathbf{N}_{v}\left(t_{k+1}, t_{k}\right)$, which contains the gyro process noise contributions to the attitude propagation errors, has moved from the bottom three rows (the $M^{L} \Delta \varphi$ rows) of Eq. (47) to the top three rows (the $\boldsymbol{\delta} \boldsymbol{\vartheta}$ rows) in Eq. (58). This is characteristic of the dynamic-model replacement mode. This mode takes the RIG data very seriously, possibly too seriously, believing that the only error in the RIG data is the output noise $\mathbf{v}_{e}$. An estimator using rate gyros in the dynamic-model replacement mode has nothing analogous to the $M^{L} \Delta \boldsymbol{\varphi}$ rows, so it puts the gyro process noise in the only available place, the $\boldsymbol{\delta} \vartheta$ rows. That estimator takes the gyro data equally seriously, if not more so.

The error-covariance propagates according to

$$
P_{k+1}=\Phi_{\mathrm{eff}}\left(t_{k+1}, t_{k}\right) P_{k} \Phi_{\mathrm{eff}}^{T}\left(t_{k+1}, t_{k}\right)+G\left(t_{k+1}, t_{k}\right) Q G^{T}\left(t_{k+1}, t_{k}\right)
$$


The process noise covariance $Q$ is given by

$$
\begin{gathered}
Q=E\left\{\left[\begin{array}{c}
-\mathbf{N}_{v}\left(t_{k+1}, t_{k}\right)-\mathbf{v}_{e} \\
\mathbf{N}_{u}\left(t_{k+1}, t_{k}\right) \\
-\mathbf{v}_{e}
\end{array}\right]\left[\begin{array}{c}
-\mathbf{N}_{v}\left(t_{k+1}, t_{k}\right)-\mathbf{v}_{e} \\
\mathbf{N}_{u}\left(t_{k+1}, t_{k}\right) \\
-\mathbf{v}_{e}
\end{array}\right]^{T}\right\} \\
=\left[\begin{array}{ccc}
Q_{v} \delta t+\frac{1}{3} Q_{u} \delta t^{3}+Q_{e} & -\frac{1}{2} Q_{u} \delta t^{2} & Q_{e} \\
-\frac{1}{2} Q_{u} \delta t^{2} & Q_{u} \delta t & 0_{3 \times 3} \\
Q_{e} & 0_{3 \times 3} & Q_{e}
\end{array}\right]
\end{gathered}
$$

These equations are the three-axis equivalents of Eqs. (23) and (24). Using the notation $\tilde{Q}_{e} \triangleq M^{L} Q_{e}\left(M^{L}\right)^{T}$ and

$$
P=\left[\begin{array}{ccc}
P_{\vartheta \vartheta} & P_{\beta \vartheta}^{T} & P_{\varphi \vartheta}^{T} \\
P_{\beta \vartheta} & P_{\beta \beta} & P_{\beta \varphi} \\
P_{\varphi \vartheta} & P_{\beta \varphi}^{T} & P_{\varphi \varphi}
\end{array}\right]=\left[\begin{array}{ccc}
\tilde{P} & P_{\varphi \vartheta}^{T} \\
& P_{\beta \varphi} \\
P_{\varphi \vartheta} & P_{\beta \varphi}^{T} & P_{\varphi \varphi}
\end{array}\right]
$$

where

$$
\tilde{P} \triangleq\left[\begin{array}{ll}
P_{\vartheta \vartheta} & P_{\beta \vartheta}^{T} \\
P_{\beta \vartheta} & P_{\beta \beta}
\end{array}\right]
$$

Eq. (59) can be written as

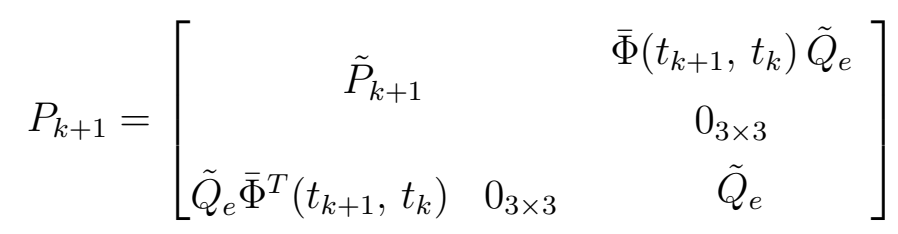

with

$$
\tilde{P}_{k+1}=\tilde{\Phi}\left(t_{k+1}, t_{k}\right)\left[\tilde{P}_{k}+\Delta \tilde{P}\left(t_{k+1}, t_{k}\right)\right] \tilde{\Phi}^{T}\left(t_{k+1}, t_{k}\right)+\tilde{Q}\left(t_{k+1}, t_{k}\right)
$$

where $\tilde{\Phi}\left(t_{k+1}, t_{k}\right)$ and $\tilde{Q}\left(t_{k+1}, t_{k}\right)$ are the upper left $6 \times 6$ corners of $\Phi_{\text {eff }}\left(t_{k+1}, t_{k}\right)$ and 
$G\left(t_{k+1}, t_{k}\right) Q G^{T}\left(t_{k+1}, t_{k}\right)$, respectively, and

$$
\begin{aligned}
\Delta \tilde{P}\left(t_{k+1}, t_{k}\right) & =\tilde{\Phi}^{-1}\left(t_{k+1}, t_{k}\right)\left[\begin{array}{cc}
\bar{\Phi}\left(t_{k+1}, t_{k}\right) P_{\varphi \varphi_{k}} \bar{\Phi}^{T}\left(t_{k+1}, t_{k}\right) & 0_{3 \times 3} \\
0_{3 \times 3} & 0_{3 \times 3}
\end{array}\right] \tilde{\Phi}^{-T}\left(t_{k+1}, t_{k}\right) \\
& -\left[\begin{array}{cc}
P_{\varphi \vartheta_{k}}^{T} \bar{\Phi}^{T}\left(t_{k+1}, t_{k}\right) & 0_{3 \times 3} \\
P_{\beta \varphi_{k}} \bar{\Phi}^{T}\left(t_{k+1}, t_{k}\right) & 0_{3 \times 3}
\end{array}\right] \tilde{\Phi}^{-T}\left(t_{k+1}, t_{k}\right) \\
& -\tilde{\Phi}^{-1}\left(t_{k+1}, t_{k}\right)\left[\begin{array}{cc}
\bar{\Phi}\left(t_{k+1}, t_{k}\right) P_{\varphi \vartheta_{k}} & \bar{\Phi}\left(t_{k+1}, t_{k}\right) P_{\beta \varphi_{k}}^{T} \\
0_{3 \times 3} & 0_{3 \times 3}
\end{array}\right]
\end{aligned}
$$

Equations (46) and (52), and $\left[\hat{\mathbf{e}}_{k+1, k} \times\right]^{3}=-\left[\hat{\mathbf{e}}_{k+1, k} \times\right]$ show that $\Phi_{\vartheta \vartheta}\left(t_{k+1}, t_{k}\right)$ is orthogonal and $\Phi_{\vartheta \vartheta}^{T}\left(t_{k+1}, t_{k}\right) \bar{\Phi}\left(t_{k+1}, t_{k}\right)=\bar{\Phi}^{T}\left(t_{k+1}, t_{k}\right)$, so

$$
\tilde{\Phi}^{-1}\left(t_{k+1}, t_{k}\right)=\left[\begin{array}{cc}
\Phi_{\vartheta \vartheta}^{T}\left(t_{k+1}, t_{k}\right) & \bar{\Phi}^{T}\left(t_{k+1}, t_{k}\right) \delta t \\
0_{3 \times 3} & I_{3}
\end{array}\right]
$$

Equation (65) can now be re-expressed as

$$
\Delta \tilde{P}\left(t_{k+1}, t_{k}\right)=\left[\begin{array}{cc}
\Delta \tilde{P}_{\vartheta \vartheta}\left(t_{k+1}, t_{k}\right) & -\bar{\Phi}^{T}\left(t_{k+1}, t_{k}\right) P_{\beta \varphi_{k}}^{T} \\
-P_{\beta \varphi_{k}} \bar{\Phi}\left(t_{k+1}, t_{k}\right) & 0_{3 \times 3}
\end{array}\right]
$$

where

$$
\Delta \tilde{P}_{\vartheta \vartheta}\left(t_{k+1}, t_{k}\right) \triangleq \bar{\Phi}^{T}\left(t_{k+1}, t_{k}\right) P_{\varphi \varphi_{k}} \bar{\Phi}\left(t_{k+1}, t_{k}\right)-P_{\varphi \vartheta_{k}}^{T} \bar{\Phi}\left(t_{k+1}, t_{k}\right)-\bar{\Phi}^{T}\left(t_{k+1}, t_{k}\right) P_{\varphi \vartheta_{k}}
$$

Equations (63), (64), (67), and (68) are computationally less expensive than Eq. (59), and they also serve better to show the relation between the RIG formulation and the conventional formulation.

\section{Cancellation of Gyro Measurement Output Noise}

The one-dimensional case shown previously has the property that the gyro measurement output noise does not propagate forward in time, which is to say that the output noise added at one gyro propagation step exactly cancels out in the next propagation step. This is reasonable because a measurement error in one RIG output leads to an incremental angle error at that step but also to an incremental angle error of equal magnitude but opposite sign at the next step. It is useful to see if this property holds in the three-dimensional case. Consider two successive gyro propagation steps, from $t_{k}$ to $t_{k+1}$ and from $t_{k+1}$ to $t_{k+2}$. 
Equation (63) states that $P_{\beta \varphi_{k+1}}=0_{3 \times 3}, P_{\varphi \vartheta_{k+1}}=\tilde{Q}_{e} \bar{\Phi}^{T}\left(t_{k+1}, t_{k}\right)$, and $P_{\varphi \varphi_{k+1}}=\tilde{Q}_{e}$, so Eqs. (63), (65), and (67) give

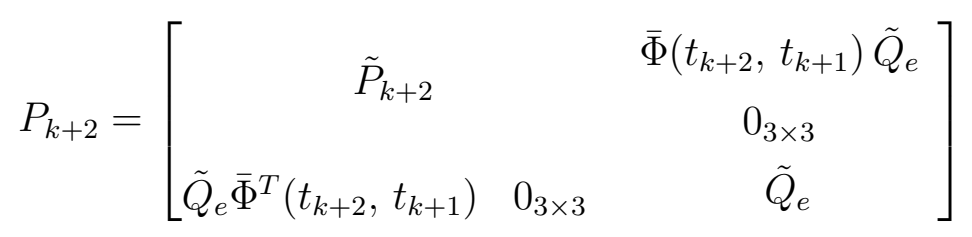

with

$$
\tilde{P}_{k+2}=\tilde{\Phi}\left(t_{k+2}, t_{k+1}\right)\left[\tilde{P}_{k+1}+\Delta \tilde{P}\left(t_{k+2}, t_{k+1}\right)\right] \tilde{\Phi}^{T}\left(t_{k+2}, t_{k+1}\right)+\tilde{Q}\left(t_{k+2}, t_{k+1}\right)
$$

and

$$
\begin{aligned}
\Delta \tilde{P}\left(t_{k+2}, t_{k+1}\right) & =\left[\begin{array}{cc}
\Delta \tilde{P}_{\vartheta \vartheta}\left(t_{k+2}, t_{k+1}\right) & -\bar{\Phi}^{T}\left(t_{k+2}, t_{k+1}\right) P_{\beta \varphi_{k+1}}^{T} \\
-P_{\beta \varphi_{k+1}} \bar{\Phi}\left(t_{k+2}, t_{k+1}\right) & 0_{3 \times 3}
\end{array}\right] \\
& =\left[\begin{array}{cc}
\Delta \tilde{P}_{\vartheta \vartheta}\left(t_{k+2}, t_{k+1}\right) & 0_{3 \times 3} \\
0_{3 \times 3} & 0_{3 \times 3}
\end{array}\right]
\end{aligned}
$$

where

$$
\begin{aligned}
\Delta \tilde{P}_{\vartheta \vartheta}\left(t_{k+2}, t_{k+1}\right) & =\bar{\Phi}^{T}\left(t_{k+2}, t_{k+1}\right) P_{\varphi \varphi_{k+1}} \bar{\Phi}\left(t_{k+2}, t_{k+1}\right)-P_{\varphi \vartheta_{k+1}}^{T} \bar{\Phi}\left(t_{k+2}, t_{k+1}\right) \\
& -\bar{\Phi}^{T}\left(t_{k+2}, t_{k+1}\right) P_{\varphi \vartheta_{k+1}} \\
& =\bar{\Phi}^{T}\left(t_{k+2}, t_{k+1}\right) \tilde{Q}_{e} \bar{\Phi}\left(t_{k+2}, t_{k+1}\right)-\bar{\Phi}\left(t_{k+1}, t_{k}\right) \tilde{Q}_{e} \bar{\Phi}\left(t_{k+2}, t_{k+1}\right) \\
& -\bar{\Phi}^{T}\left(t_{k+2}, t_{k+1}\right) \tilde{Q}_{e} \bar{\Phi}^{T}\left(t_{k+1}, t_{k}\right) \\
& =\left[\bar{\Phi}^{T}\left(t_{k+2}, t_{k+1}\right)-\bar{\Phi}\left(t_{k+1}, t_{k}\right)\right] \tilde{Q}_{e}\left[\bar{\Phi}\left(t_{k+2}, t_{k+1}\right)-\bar{\Phi}^{T}\left(t_{k+1}, t_{k}\right)\right] \\
& -\bar{\Phi}\left(t_{k+1}, t_{k}\right) \tilde{Q}_{e} \bar{\Phi}^{T}\left(t_{k+1}, t_{k}\right)
\end{aligned}
$$

The $\tilde{Q}_{e}$ terms in Eq. (69) and the $\tilde{Q}\left(t_{k+2}, t_{k+1}\right)$ term in Eq. (70) contain RIG output noise only from $t_{k+2}$, so RIG output noise from $t_{k+1}$ can find its way into $P_{k+2}$ only through $\tilde{P}_{k+1}$ and $\Delta \tilde{P}\left(t_{k+2}, t_{k+1}\right)$. The only contribution to $\tilde{P}_{k+1}$ from RIG output noise at $t_{k+1}$ is a term $\bar{\Phi}\left(t_{k+1}, t_{k}\right) \tilde{Q}_{e} \bar{\Phi}^{T}\left(t_{k+1}, t_{k}\right)$ in the upper left $3 \times 3$ corner coming from the same corner of $\tilde{Q}\left(t_{k+1}, t_{k}\right)$, and this term is exactly cancelled by the $-\bar{\Phi}\left(t_{k+1}, t_{k}\right) \tilde{Q}_{e} \bar{\Phi}^{T}\left(t_{k+1}, t_{k}\right)$ term in $\Delta \tilde{P}_{\vartheta \vartheta}\left(t_{k+2}, t_{k+1}\right)$. Thus the contribution to $P_{k+2}$ from RIG output noise at $t_{k+1}$ vanishes if 
and only if

$$
\begin{aligned}
0_{3 \times 3} & =\bar{\Phi}^{T}\left(t_{k+2}, t_{k+1}\right)-\bar{\Phi}\left(t_{k+1}, t_{k}\right) \\
& =\frac{1-\cos \left(\hat{\psi}_{k+2, k+1}\right)}{\hat{\psi}_{k+2, k+1}}\left[\hat{\mathbf{e}}_{k+2, k+1} \times\right]+\frac{\hat{\psi}_{k+2, k+1}-\sin \left(\hat{\psi}_{k+2, k+1}\right)}{\hat{\psi}_{k+2, k+1}}\left[\hat{\mathbf{e}}_{k+2, k+1} \times\right]^{2} \\
& +\frac{1-\cos \left(\hat{\psi}_{k+1, k}\right)}{\hat{\psi}_{k+1, k}}\left[\hat{\mathbf{e}}_{k+1, k} \times\right]-\frac{\hat{\psi}_{k+1, k}-\sin \left(\hat{\psi}_{k+1, k}\right)}{\hat{\psi}_{k+1, k}}\left[\hat{\mathbf{e}}_{k+1, k} \times\right]^{2}
\end{aligned}
$$

Examination of Eq. (73) shows that this cancellation happens if and only if $\hat{\mathbf{e}}_{k+2, k+1}=$ $\hat{\mathbf{e}}_{k+1, k} \triangleq \hat{\mathbf{e}}$ and either $\hat{\psi}_{k+2, k+1}=-\hat{\psi}_{k+1, k}$ or $\hat{\psi}_{k+1, k}=2 \pi n$ and $\hat{\psi}_{k+2, k+1}=2 \pi m$, where $n$ and $m$ are nonzero integers. The matrices $\left[\hat{\boldsymbol{\psi}}_{k+1, k} \otimes\right]$ and $\left[\hat{\boldsymbol{\psi}}_{k+2, k+1} \otimes\right]$ commute in both of these cases, so Eq. (36) gives

$$
\begin{aligned}
\hat{\mathbf{q}}_{k+2} & =\exp \left(\frac{1}{2}\left[\hat{\boldsymbol{\psi}}_{k+2, k+1} \otimes\right]\right) \hat{\mathbf{q}}_{k+1} \\
& =\exp \left(\frac{1}{2}\left[\hat{\boldsymbol{\psi}}_{k+2, k+1} \otimes\right]\right)\left\{\exp \left(\frac{1}{2}\left[\hat{\boldsymbol{\psi}}_{k+1, k} \otimes\right]\right) \hat{\mathbf{q}}_{k}\right\} \\
& =\exp \left(\frac{1}{2}\left[\left(\hat{\boldsymbol{\psi}}_{k+2, k+1}+\hat{\boldsymbol{\psi}}_{k+1, k}\right) \otimes\right]\right) \hat{\mathbf{q}}_{k}
\end{aligned}
$$

The case $\mathbf{0}_{3}=\hat{\boldsymbol{\psi}}_{k+2, k+1}+\hat{\boldsymbol{\psi}}_{k+1, k}=\int_{t_{k}}^{t_{k+1}} \hat{\boldsymbol{\omega}}(\tau) d \tau$, which gives $\hat{\mathbf{q}}_{k+2}=\hat{\mathbf{q}}_{k}$, includes the special case that $\hat{\boldsymbol{\omega}}=\mathbf{0}_{3}$ over the entire span from $t_{k}$ to $t_{k+2}$. It is not surprising that the output error does not propagate forward if $\hat{\boldsymbol{\omega}}=\mathbf{0}_{3}$, because the estimator has no coupling between the coordinate axes in this case, so the three-dimensional case looks like three independent single-axis cases for which it is known that the cancellation is exact. The less likely case that $\hat{\psi}_{k+1, k}=2 \pi n \hat{\mathbf{e}}$ and $\hat{\psi}_{k+2, k+1}=2 \pi m \hat{\mathbf{e}}$ gives $\hat{\mathbf{q}}_{k+2}=(-1)^{n+m} \hat{\mathbf{q}}_{k}$, so the attitude matrices at $t_{k}$ and $t_{k+2}$ are identical in all the three-axis cases for which the cancellation is exact.

If $\hat{\mathbf{e}}_{k+2, k+1}=\hat{\mathbf{e}}_{k+1, k}$ but $\hat{\psi}_{k+1, k}$ and $\hat{\psi}_{k+2, k+1}$ do not satisfy either of the conditions for cancellation specified below Eq. (73), the contribution to $P_{k+2}$ from RIG output noise at $t_{k+1}$ does not vanish because

$$
\begin{aligned}
\bar{\Phi}^{T}\left(t_{k+2}, t_{k+1}\right)-\bar{\Phi}\left(t_{k+1}, t_{k}\right) & =\left[\frac{1-\cos \left(\hat{\psi}_{k+2, k+1}\right)}{\hat{\psi}_{k+2, k+1}}+\frac{1-\cos \left(\hat{\psi}_{k+1, k}\right)}{\hat{\psi}_{k+1, k}}\right][\hat{\mathbf{e}} \times] \\
& -\left[\frac{\sin \left(\hat{\psi}_{k+2, k+1}\right)}{\hat{\psi}_{k+2, k+1}}-\frac{\sin \left(\hat{\psi}_{k+1, k}\right)}{\hat{\psi}_{k+1, k}}\right][\hat{\mathbf{e}} \times]^{2} \neq 0_{3 \times 3}
\end{aligned}
$$

The lack of cancellation in this case is somewhat surprising, because the matrices $\left[\hat{\boldsymbol{\psi}}_{k+1, k} \otimes\right]$ 
and $\left[\hat{\boldsymbol{\psi}}_{k+2, k+1} \otimes\right]$ commute, Eq. (74) holds, and Eq. (37) gives

$$
\hat{\mathbf{q}}_{k+2}=\exp \left(\frac{1}{2}\left[M^{L}\left(\hat{\boldsymbol{\varphi}}_{k+2}-\hat{\boldsymbol{\varphi}}_{k}-2 \hat{\boldsymbol{\beta}} \delta t\right) \otimes\right]\right) \hat{\mathbf{q}}_{k}
$$

with the RIG output at time $t_{k+1}$ cancelling out. Equation (75) reveals two interesting properties, though. The first is that the contribution of RIG output noise at $t_{k+1}$ to $P_{\vartheta \vartheta_{k+2}}$, $P_{\beta \vartheta_{k+2}}$, and $P_{\beta \beta_{k+2}}$ in this case is entirely in the plane perpendicular to $\hat{\mathbf{e}}$. This is consistent with the observation that if $\hat{\boldsymbol{\omega}}$ is always along a fixed axis, this axis decouples dynamically from the other two axes, so estimation of the rotation about this axis is just like the onedimensional case for which RIG output noise does not propagate forward in time. The dynamics of the two axes perpendicular to $\hat{\boldsymbol{\omega}}$ are coupled by the rotation, though; and the fact that the contribution of RIG output noise at $t_{k+1}$ to the covariance at $t_{k+2}$ is in the plane perpendicular to $\hat{\boldsymbol{\omega}}$ may help to explain why it vanishes only if the attitude matrices at $t_{k}$ and $t_{k+2}$ are identical. The second interesting property of Eq. (75) is that the contribution

of RIG output noise at $t_{k+1}$ to the covariance at $t_{k+2}$ is of order $\left(\hat{\psi}_{k+2, k+1}+\hat{\psi}_{k+1, k}\right)^{2}$ for small rotations.

Cancellation is not exact in the general case, but there is near-cancellation for small rotations, and the fundamental function of the additional three components of the state vector in the RIG formulation is to ensure this cancellation or near-cancellation.

\section{Angular Rate Estimate}

As was observed below Eq (32), the angular rate is not part of the state vector of this estimator. The rate is an important quantity of interest, however, and is typically used in a controller. Rate-integrating gyros do not output an instantaneous rate measurement, but they can provide an estimate of the average rate between times $t_{k}$ and $t_{k+1}$. Equation (35) with $\hat{\varphi}_{k+1}=\tilde{\varphi}_{k+1}$, gives

$$
\hat{\boldsymbol{\omega}}_{k+1, k}=\frac{1}{\delta t} M^{L}\left(\tilde{\boldsymbol{\varphi}}_{k+1}-\hat{\boldsymbol{\varphi}}_{k}-\hat{\boldsymbol{\beta}} \delta t\right)
$$

Equation (32) gives the true average rate over this time interval as

$$
\begin{aligned}
\boldsymbol{\omega}_{k+1, k} & =\frac{1}{\delta t} \int_{t_{k}}^{t_{k+1}} \boldsymbol{\omega}(\tau) d \tau=\frac{1}{\delta t} M^{L}\left\{\boldsymbol{\varphi}_{k+1}-\boldsymbol{\varphi}_{k}-\int_{t_{k}}^{t_{k+1}}\left[\boldsymbol{\beta}(\tau)+\boldsymbol{\eta}_{v}(\tau)\right] d \tau\right\} \\
& =\frac{1}{\delta t} M^{L}\left[\boldsymbol{\varphi}_{k+1}-\boldsymbol{\varphi}_{k}-\boldsymbol{\beta}_{k} \delta t-\mathbf{N}_{v}\left(t_{k+1}, t_{k}\right)\right]
\end{aligned}
$$


The error in the angular rate estimate is

$$
\begin{aligned}
\Delta \boldsymbol{\omega}_{k+1, k} & =\boldsymbol{\omega}_{k+1, k}-\hat{\boldsymbol{\omega}}_{k+1, k} \\
& =\frac{1}{\delta t} M^{L}\left[-\mathbf{v}_{e}-\Delta \boldsymbol{\varphi}_{k}-\Delta \boldsymbol{\beta}_{k} \delta t-\mathbf{N}_{v}\left(t_{k+1}, t_{k}\right)\right]
\end{aligned}
$$

The covariance of the angular rate error, $P_{\omega \omega_{k+1}} \triangleq E\left\{\Delta \boldsymbol{\omega}_{k+1, k} \Delta \boldsymbol{\omega}_{k+1, k}^{T}\right\}$, is given by

$$
P_{\omega \omega_{k+1}}=\frac{1}{\delta t^{2}}\left(\tilde{Q}_{e}+P_{\varphi \varphi_{k}}\right)+\frac{1}{\delta t}\left(\tilde{Q}_{v}+P_{\varphi \beta_{k}}+P_{\varphi \beta_{k}}^{T}\right)+P_{\beta \beta_{k}}+\frac{1}{3} \tilde{Q}_{u} \delta t
$$

where $\tilde{Q}_{u} \triangleq M^{L} Q_{u}\left(M^{L}\right)^{T}$ and $\tilde{Q}_{v} \triangleq M^{L} Q_{v}\left(M^{L}\right)^{T}$. The Appendix contains details useful in this derivation. Equation (63) gives some simplifications if $P_{k}$ is the covariance immediately following a gyro propagation.

\section{Gyro Output Matrix Inverse}

The left inverse $M^{L}$ is now discussed. This is the usual inverse if there are only three gyros, leaving no opportunity to assign weights to the gyro measurements. With more than three gyros, the left inverse can be written as

$$
M^{L}=\left(M^{T} W M\right)^{-1} M^{T} W
$$

where the symmetric positive semi-definite weight matrix $W$ must be chosen so that the inverse in Eq. (81) exists. The simplest choice is $W=I_{n}$, but it might be better to choose gyro weights inversely proportional to their error variances. The form of the upper left

$3 \times 3$ corner of $Q$ suggests that $W=\left(Q_{v} \delta t+\frac{1}{3} Q_{u} \delta t^{3}+Q_{e}\right)^{-1}$ be chosen. The components of the diagonal matrices $Q_{v}, Q_{u}$, and $Q_{e}$ can be different on the different axes, but the more common case is that all the gyros have identical noise characteristics, so $Q_{v}=\sigma_{v}^{2} I_{n}$, $Q_{u}=\sigma_{u}^{2} I_{n}$, and $Q_{e}=\sigma_{e}^{2} I_{n}$. In this case, the above choice for $W$ makes it a multiple of the identity matrix, and it is clear from Eq. (81) that choosing $W$ to be any multiple of the identity is equivalent to choosing it to be equal to the identity matrix. If all the gyros have identical noise characteristics, then, there is no reason to choose $W$ to be anything other than the identity matrix.

\section{Measurement Update Equations}

The measurement update equations generally follow the the conventional MEKF presented in Ref. [7]. This section presents only the special features of the RIG-based estimator with the $m$-component attitude measurement model of Eq. (9). The detailed equations can be found in Table 1. 
The state estimate and covariance prior to the measurement update are denoted by

$$
\hat{\mathbf{x}}^{-}=\left[\begin{array}{c}
\hat{\mathbf{q}}^{-} \\
M^{L} \hat{\boldsymbol{\beta}}^{-} \\
M^{L} \hat{\boldsymbol{\varphi}}^{-}
\end{array}\right]
$$

and $\hat{P}^{-}$respectively. These can follow either a gyro propagation or an attitude measurement update with no intervening propagation step. The sensitivity matrix for the measurement vector of Eq. (9) is

$$
H_{k}=\left[\begin{array}{lll}
\tilde{H}_{k} & 0_{3 N \times 3} & 0_{3 N \times 3}
\end{array}\right]
$$

with $[7,11]$

$$
\left.\tilde{H}_{k} \triangleq\left[\begin{array}{c}
{\left[A\left(\hat{\mathbf{q}}^{-}\right) \mathbf{r}_{1} \times\right]} \\
\vdots \\
{\left[A\left(\hat{\mathbf{q}}^{-}\right) \mathbf{r}_{N} \times\right]}
\end{array}\right]\right|_{t_{k}}
$$

The Kalman gain and the covariance update are given by

$$
\begin{gathered}
K_{k}=P_{k}^{-} H_{k}^{T}\left(H_{k} P_{k}^{-} H_{k}^{T}+R_{k}\right)^{-1}=P_{k}^{-}\left[\begin{array}{cc}
\tilde{H}_{k} & 0_{3 N \times 6}
\end{array}\right]^{T}\left(\tilde{H}_{k} P_{\vartheta \vartheta_{k}}^{-} \tilde{H}_{k}^{T}+R_{k}\right)^{-1} \\
P_{k}^{+}=\left(I_{9}-K_{k}\left[\begin{array}{cc}
\tilde{H}_{k} & 0_{3 N \times 6}
\end{array}\right]\right) P_{k}^{-}
\end{gathered}
$$

where the superscript + indicates a post-update quantity.

\section{Gyro Measurement Output Noise-Free Case}

This subsection compares the filter in the limiting case of negligibly small gyro measurement output noise with the conventional MEKF presented in Ref. [7]. When $\tilde{Q}_{e}=0_{3 \times 3}$, the rightmost three columns and the bottom three rows of the $9 \times 9$ covariance matrix $P_{k}$ are identically zero, as is the matrix $\Delta \tilde{P}\left(t_{k+1}, t_{k}\right)$. The matrix $\tilde{\Phi}\left(t_{k+1}, t_{k}\right)$ is the same as the matrix $\Phi_{k}$ in the traditional MEKF, so the covariance propagation is the same as the traditional MEKF except for a different computation of $Q$. For consistency with Ref. [7], assume that there are three gyros with identical noise characteristics and with $M=I_{3}$, so $\tilde{Q}_{v}=\sigma_{v}^{2} I_{3}$, and $\tilde{Q}_{v}=\sigma_{u}^{2} I_{3}$. The conventional MEKF, with notation changed to agree with this paper, gives the process noise covariance matrix as [7]

$$
Q_{k}=\left[\begin{array}{ll}
Q_{11_{k}} & Q_{12_{k}} \\
Q_{12_{k}}^{T} & Q_{22_{k}}
\end{array}\right]
$$


with

$$
\begin{gathered}
Q_{22_{k}}=\left(\sigma_{u}^{2} \delta t\right) I_{3} \\
Q_{12_{k}}=-\frac{1}{2} \sigma_{u}^{2} \delta t^{2}\left\{I_{3}-2 \frac{\hat{\psi}_{k+1, k}-\sin \left(\hat{\psi}_{k+1, k}\right)}{\hat{\psi}_{k+1, k}^{2}}\left[\hat{\mathbf{e}}_{k+1, k} \times\right]\right. \\
\left.+\frac{\hat{\psi}_{k+1, k}^{2}+2 \cos \left(\hat{\psi}_{k+1, k}\right)-2}{\hat{\psi}_{k+1, k}^{2}}\left[\hat{\mathbf{e}}_{k+1, k} \times\right]^{2}\right\} \\
\approx-\frac{1}{2} \sigma_{u}^{2} \delta t^{2}\left\{I_{3}-\frac{1}{3}\left[\hat{\boldsymbol{\psi}}_{k+1, k} \times\right]+\frac{1}{12}\left[\hat{\boldsymbol{\psi}}_{k+1, k} \times\right]^{2}\right\}
\end{gathered}
$$

and

$$
\begin{aligned}
Q_{11_{k}} & =\left(\sigma_{v}^{2} \delta t\right) I_{3}+\frac{1}{3} \sigma_{u}^{2} \delta t^{3}\left\{I_{3}-\frac{6 \hat{\psi}_{k+1, k}-6 \sin \left(\hat{\psi}_{k+1, k}\right)-\hat{\psi}_{k+1, k}^{3}}{\hat{\psi}_{k+1, k}^{3}}\left[\hat{\mathbf{e}}_{k+1, k} \times\right]^{2}\right\} \\
& \approx\left(\sigma_{v}^{2} \delta t\right) I_{3}+\frac{1}{3} \sigma_{u}^{2} \delta t^{3}\left\{I_{3}+\frac{1}{20}\left[\hat{\boldsymbol{\psi}}_{k+1, k} \times\right]^{2}\right\}
\end{aligned}
$$

The approximations are the lowest-order terms in $\hat{\psi}_{k+1, k}$.

The corresponding matrix in the RIG case using $\bar{\Phi}\left(t_{k+1}, t_{k}\right)$ is

$$
\tilde{Q}_{k+1, k}=\left[\begin{array}{ll}
\tilde{Q}_{11} & \tilde{Q}_{12} \\
\tilde{Q}_{12}^{T} & \tilde{Q}_{22}
\end{array}\right]
$$

with

$$
\begin{aligned}
\tilde{Q}_{22}=\left(\sigma_{u}^{2} \delta t\right) I_{3} \\
\tilde{Q}_{12}=-\frac{1}{2}\left(\sigma_{u}^{2} \delta t^{2}\right) \bar{\Phi}\left(t_{1}, t_{0}\right) \\
=-\frac{1}{2} \sigma_{u}^{2} \delta t^{2}\left\{I_{3}-\frac{1-\cos \left(\hat{\psi}_{k+1, k}\right)}{\hat{\psi}_{k+1, k}}\left[\hat{\mathbf{e}}_{k+1, k} \times\right]+\frac{\hat{\psi}_{k+1, k}-\sin \left(\hat{\psi}_{k+1, k}\right)}{\hat{\psi}_{k+1, k}}\left[\hat{\mathbf{e}}_{k+1, k} \times\right]^{2}\right\} \\
\approx-\frac{1}{2} \sigma_{u}^{2} \delta t^{2}\left\{I_{3}-\frac{1}{2}\left[\hat{\boldsymbol{\psi}}_{k+1, k} \times\right]+\frac{1}{6}\left[\hat{\boldsymbol{\psi}}_{k+1, k} \times\right]^{2}\right\}
\end{aligned}
$$

and

$$
\begin{aligned}
\tilde{Q}_{11} & =\left(\sigma_{v}^{2} \delta t+\frac{1}{3} \sigma_{u}^{2} \delta t^{3}\right) \bar{\Phi}\left(t_{1}, t_{0}\right) \bar{\Phi}^{T}\left(t_{1}, t_{0}\right) \\
& =\left(\sigma_{v}^{2} \delta t+\frac{1}{3} \sigma_{u}^{2} \delta t^{3}\right)\left\{I_{3}+\frac{\hat{\psi}_{k+1, k}^{2}+2 \cos \left(\hat{\psi}_{k+1, k}\right)-2}{\hat{\psi}_{k+1, k}^{2}}\left[\hat{\mathbf{e}}_{k+1, k} \times\right]^{2}\right\} \\
& \approx\left(\sigma_{v}^{2} \delta t+\frac{1}{3} \sigma_{u}^{2} \delta t^{3}\right)\left\{I_{3}+\frac{1}{12}\left[\hat{\boldsymbol{\psi}}_{k+1, k} \times\right]^{2}\right\}
\end{aligned}
$$


The process noise covariance the same in lowest (zeroth) order in $\hat{\psi}_{k+1, k}$ but $Q_{11}$ and $Q_{12}$ differ in higher orders. The zeroth order approximation is generally adequate in practice, as explained in Ref. [7]. The similarity of the two approaches is misleading, because they compute the incremental angle $\hat{\boldsymbol{\psi}}_{k+1, k} \triangleq \int_{t_{k}}^{t_{k+1}} \hat{\boldsymbol{\omega}}(\tau) d \tau$ differently, in principle. In the conventional method, the rate gyros are assumed to output a continuous rate $\hat{\boldsymbol{\omega}}(t)$, which is integrated by the estimator. The RIG estimator, in contrast, computes $\hat{\boldsymbol{\psi}}_{k+1, k}$ as a finite difference of $\tilde{\varphi}_{k}$ values output by the RIGs at discrete times.

\section{Reduced Rate-Integrating Gyro-Based Kalman Filter}

This section presents the reduced-order RIG-based MEKF. In this case the integral of the angular rate is estimated using

$$
\hat{\boldsymbol{\psi}}_{k+1, k} \triangleq \int_{t_{k}}^{t_{k+1}} \hat{\boldsymbol{\omega}}(\tau) d \tau=M^{L}\left(\tilde{\boldsymbol{\varphi}}_{k+1}-\tilde{\boldsymbol{\varphi}}_{k}-\hat{\boldsymbol{\beta}} \delta t\right)
$$

The quantity $\varphi$ can now be removed from the state vector, giving the following sevencomponent "global" truth state vector and six-component "local" error-state vector, respectively:

$$
\mathbf{x}^{r}=\left[\begin{array}{c}
\mathbf{q} \\
M^{L} \boldsymbol{\beta}
\end{array}\right], \quad \Delta \mathbf{x}^{r}=\left[\begin{array}{c}
\boldsymbol{\delta} \boldsymbol{\vartheta} \\
M^{L} \Delta \boldsymbol{\beta}
\end{array}\right]
$$

The true rate is given by Eq. (32c) using a finite-difference approximation for $\dot{\varphi}$

$$
\boldsymbol{\omega}=M^{L}\left(\frac{\boldsymbol{\varphi}_{k+1}-\boldsymbol{\varphi}_{k}}{\delta t}-\boldsymbol{\beta}-\boldsymbol{\eta}_{v}\right)
$$

This gives the attitude rate error as

$$
\Delta \boldsymbol{\omega}=M^{L}\left(\frac{-\mathbf{v}_{e_{k+1}}+\mathbf{v}_{e_{k}}}{\delta t}-\Delta \boldsymbol{\beta}-\boldsymbol{\eta}_{v}\right)
$$

Inserting this into the error dynamics of Eqs. (41a) and (41b) gives

$$
\Delta \dot{\mathbf{x}}^{r}=\left[\begin{array}{cc}
-[\hat{\boldsymbol{\omega}} \times] & -I_{3} \\
0_{3 \times 3} & 0_{3 \times 3}
\end{array}\right] \Delta \mathbf{x}^{r}+\left[\begin{array}{c}
M^{L}\left(-\mathbf{v}_{e_{k+1}}+\mathbf{v}_{e_{k}}\right) / \delta t-M^{L} \boldsymbol{\eta}_{v} \\
M^{L} \boldsymbol{\eta}_{u}
\end{array}\right]
$$


The same logic as that leading to Eq. (44) gives

$$
\begin{aligned}
\Delta \mathbf{x}_{k+1}^{r} & =\tilde{\Phi}\left(t_{k+1}, t_{k}\right) \Delta \mathbf{x}_{k}^{r}+\int_{t_{k}}^{t_{k+1}} \tilde{\Phi}\left(t_{k+1}, \tau\right)\left[\begin{array}{c}
-M^{L} \boldsymbol{\eta}_{v}(\tau) \\
M^{L} \boldsymbol{\eta}_{u}(\tau)
\end{array}\right] d \tau \\
& +\left[\begin{array}{c}
\bar{\Phi}\left(t_{k+1}, t_{k}\right) M^{L}\left(-\mathbf{v}_{e_{k+1}}+\mathbf{v}_{e_{k}}\right) \\
\mathbf{0}_{3}
\end{array}\right]
\end{aligned}
$$

The covariance of the reduced state propagates by

$$
P_{k+1}^{r}=\tilde{\Phi}\left(t_{k+1}, t_{k}\right) P_{k}^{r} \tilde{\Phi}^{T}\left(t_{k+1}, t_{k}\right)+Q^{r}
$$

The process noise covariance $Q^{r}$ is the three-axis equivalent of Eq. (29). It is the sum of independent contributions from the second and third terms on the right side of Eq. (99). The second term gives the same process noise covariance matrix as the conventional rate gyrobased estimator, which is given by Eqs. (86)-(89) if $M=I_{3}$ and the gyros have identical noise characteristics. The third term gives an additional contribution of $2 \bar{\Phi}\left(t_{k+1}, t_{k}\right) \tilde{Q}_{e} \bar{\Phi}^{T}\left(t_{k+1}, t_{k}\right)$ to $Q_{11_{k}}$.

The angular rate estimate is given by

$$
\hat{\boldsymbol{\omega}}_{k+1, k}^{r}=\frac{1}{\delta t} M^{L}\left(\tilde{\boldsymbol{\varphi}}_{k+1}-\tilde{\boldsymbol{\varphi}}_{k}-\hat{\boldsymbol{\beta}}_{k} \delta t\right)
$$

The error in this estimate is

$$
\Delta \boldsymbol{\omega}_{k+1, k}^{r}=\frac{1}{\delta t} M^{L}\left[-\mathbf{v}_{e_{k+1}}+\mathbf{v}_{e_{k}}-\Delta \boldsymbol{\beta}_{k} \delta t-\mathbf{N}_{v}\left(t_{k+1}, t_{k}\right)\right]
$$

and its error-covariance is given by

$$
P_{\omega \omega_{k+1}}^{r}=\frac{2}{\delta t^{2}} \tilde{Q}_{e}+\frac{1}{\delta t} \tilde{Q}_{v}+P_{\beta \beta_{k}}^{r}+\frac{1}{3} \tilde{Q}_{u} \delta t
$$

This is simpler than Eq. (80), but the two expressions take the same form if $P_{k}$ satisfies Eq. (63).

The attitude measurement update equations are the same as those for the full order RIG-based Kalman filter, except for obvious changes due to the reduced dimensionality of the state vector. 
Table 1. Rate-Integrating Gyro Extended Kalman Filter

\begin{tabular}{|c|c|}
\hline Initialize & $\begin{aligned} & \hat{\mathbf{x}}_{0}^{-} \triangleq {\left[\begin{array}{c}\hat{\mathbf{q}}_{0}^{-} \\
\hat{\boldsymbol{\beta}}_{0}^{-} \\
\hat{\boldsymbol{\varphi}}_{0}^{-}\end{array}\right]=\left[\begin{array}{c}\hat{\mathbf{q}}_{0} \\
\hat{\boldsymbol{\beta}}_{0} \\
\hat{\boldsymbol{\varphi}}_{0}\end{array}\right] } \\
& P_{0}^{-}=P_{0}\end{aligned}$ \\
\hline Gain & $\begin{array}{c}K_{k}=P_{k}^{-}\left[\tilde{H}_{k}\left(\hat{\mathbf{x}}_{k}^{-}\right) 0_{3 N \times 6}\right]^{T}\left[\tilde{H}_{k}\left(\hat{\mathbf{x}}_{k}^{-}\right) P_{\vartheta \vartheta_{k}}^{-} \tilde{H}_{k}^{T}\left(\hat{\mathbf{x}}_{k}^{-}\right)+R_{k}\right]^{-1} \\
\tilde{H}_{k}\left(\hat{\mathbf{x}}_{k}^{-}\right)=\left.\left[\begin{array}{c}{\left[A\left(\hat{\mathbf{q}}_{k}^{-}\right) \mathbf{r}_{1} \times\right]} \\
\vdots \\
{\left[A\left(\hat{\mathbf{q}}_{k}^{-}\right) \mathbf{r}_{N} \times\right]}\end{array}\right]\right|_{t_{k}}\end{array}$ \\
\hline Update & $\begin{array}{c}P_{k}^{+}=\left(I_{9}-K_{k}\left[\begin{array}{ll}\tilde{H}_{k}\left(\hat{\mathbf{x}}_{k}^{-}\right) & 0_{3 N \times 6}\end{array}\right]\right) P_{k}^{-} \\
\Delta \hat{\mathbf{x}}_{k}^{+} \triangleq\left[\begin{array}{c}\boldsymbol{\delta} \hat{\boldsymbol{\vartheta}}_{k}^{+} \\
\Delta \hat{\boldsymbol{\beta}}_{k}^{+} \\
\Delta \hat{\boldsymbol{\varphi}}_{k}^{+}\end{array}\right]=K_{k}\left[\tilde{\mathbf{y}}_{k}-\mathbf{h}_{k}\left(\hat{\mathbf{x}}_{k}^{-}\right)\right] \\
\mathbf{h}_{k}\left(\hat{\mathbf{x}}_{k}^{-}\right)=\left.\left[\begin{array}{c}A\left(\hat{\mathbf{q}}_{k}^{-}\right) \mathbf{r}_{1} \\
\vdots \\
A\left(\hat{\mathbf{q}}_{k}^{-}\right) \mathbf{r}_{N}\end{array}\right]\right|_{t_{k}} \\
\hat{\mathbf{q}}^{*}=\hat{\mathbf{q}}_{k}^{-}+\frac{1}{2} \Xi\left(\hat{\mathbf{q}}_{k}^{-}\right) \boldsymbol{\delta} \hat{\boldsymbol{\vartheta}}_{k}^{+} \\
\hat{\mathbf{q}}_{k}^{+}=\hat{\mathbf{q}}^{*} /\left\|\hat{\mathbf{q}}^{*}\right\| \\
M^{L} \hat{\boldsymbol{\beta}}_{k}^{+}=M^{L} \hat{\boldsymbol{\beta}}_{k}^{-}+\Delta \hat{\boldsymbol{\beta}}_{k}^{+} \\
M^{L} \hat{\boldsymbol{\varphi}}_{k}^{+}=M^{L} \hat{\boldsymbol{\varphi}}_{k}^{-}+\Delta \hat{\boldsymbol{\varphi}}_{k}^{+}\end{array}$ \\
\hline Propagation & $\begin{array}{c}\hat{\boldsymbol{\psi}}_{k+1, k}=M^{L} \tilde{\boldsymbol{\varphi}}_{k+1}-M^{L} \hat{\boldsymbol{\varphi}}_{k}-M^{L} \hat{\boldsymbol{\beta}}_{k} \delta t \\
\hat{\mathbf{q}}_{k+1}=\exp \left(\frac{1}{2}\left[\hat{\boldsymbol{\psi}}_{k+1, k} \otimes\right]\right) \hat{\mathbf{q}}_{k} \\
M^{L} \hat{\boldsymbol{\beta}}_{k+1}=M^{L} \hat{\boldsymbol{\beta}}_{k} \\
M^{L} \hat{\boldsymbol{\varphi}}_{k+1}=M^{L} \tilde{\boldsymbol{\varphi}}_{k+1} \\
P_{k+1}=\Phi_{\mathrm{eff}}\left(t_{k+1}, t_{k}\right) P_{k} \Phi_{\text {eff }}^{T}\left(t_{k+1}, t_{k}\right)+G\left(t_{k+1}, t_{k}\right) Q G^{T}\left(t_{k+1}, t_{k}\right)\end{array}$ \\
\hline
\end{tabular}

\section{Algorithm Summaries}

Table 1 shows the RIG MEKF algorithm for attitude estimation. First, the estimated quaternion, bias vector, and RIG vectors, as well as the error-covariance are initialized. The table assumes that an update occurs before any gyro propagation, but this assumption is 
not essential. The Kalman gain is computed, and the state vector and covariance matrix are updated. Note that an explicit reset operation is not needed because $\boldsymbol{\delta} \hat{\boldsymbol{\vartheta}}_{k}^{-}$is always zero in this formulation. The updated estimates and error-covariance are then propagated. It is important to realize that the sampling rate of the RIG measurement is usually higher than the sampling rate of the attitude measurement, so there may be many propagation steps between successive attitude measurements. For this reason, superscripts - or + are not shown in the propagation equations. A series of propagations begins with $\hat{\mathbf{x}}^{+}$and $P^{+}$from the previous update and ends with $\hat{\mathbf{x}}^{-}$and $P^{-}$for the following update. Table 2 shows the algorithm for the reduced-order RIG MEKF for attitude estimation. The steps are the same as the full-order RIG MEKF in Table 1.

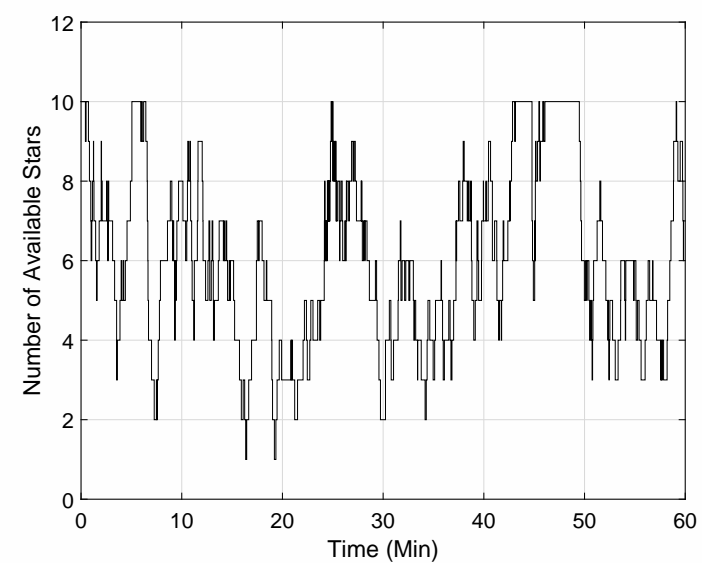

Figure 1. Availability of Stars

\section{Star Tracker Simulation}

This section shows the performance of the RIG MEKF using simulated RIG and star tracker data to estimate the attitude of an Earth-pointing spacecraft in an equatorial 350 $\mathrm{km}$ circular orbit, which is equivalent to a 91.5 minute orbital period. The spacecraft's $z$ axis is pointed in the nadir direction, the $y$-axis is pointed in the negative orbit momentum's vector, and the $x$-axis is pointed in the orbit velocity direction. The true angular velocity is given by $\boldsymbol{\omega}(t)=\left[\begin{array}{lll}0 & -1.11445 \times 10^{-3} & 0\end{array}\right]^{T} \mathrm{rad} / \mathrm{sec}$.

The star tracker is not assumed to output a quaternion, but to return unit vector observations in the body frame of individual stars that are simulated by

$$
\tilde{\mathbf{b}}_{i}=\frac{1}{\sqrt{1+\tilde{\alpha}_{i}^{2}+\tilde{\beta}_{i}^{2}}}\left[\begin{array}{c}
-\tilde{\alpha}_{i} \\
-\tilde{\beta}_{i} \\
1
\end{array}\right]
$$


Table 2. Reduced Rate-Integrating Gyro Extended Kalman Filter

\begin{tabular}{|c|c|}
\hline Initialize & $\begin{array}{c}\hat{\mathbf{x}}_{0}^{r-\triangleq}\left[\begin{array}{c}\hat{\mathbf{q}}_{0}^{-} \\
\hat{\boldsymbol{\beta}}_{0}^{-}\end{array}\right]=\left[\begin{array}{c}\hat{\mathbf{q}}_{0} \\
\hat{\boldsymbol{\beta}}_{0}\end{array}\right] \\
P_{0}^{r-}=P_{0}^{r}\end{array}$ \\
\hline Gain & $\begin{array}{c}K_{k}^{r}=P_{k}^{r-}\left[\begin{array}{ll}\tilde{H}_{k}\left(\hat{\mathbf{x}}_{k}^{r-}\right) & 0_{3 N \times 3}\end{array}\right]^{T}\left[\begin{array}{l}\tilde{H}_{k}\left(\hat{\mathbf{x}}_{k}^{r-}\right) P_{\vartheta \vartheta}^{r-} \tilde{H}_{k}^{T}\left(\hat{\mathbf{x}}_{k}^{r-}\right)+R_{k}\end{array}\right]^{-1} \\
\tilde{H}_{k}\left(\hat{\mathbf{x}}_{k}^{r-}\right)=\left.\left[\begin{array}{c}{\left[A\left(\hat{\mathbf{q}}_{k}^{-}\right) \mathbf{r}_{1} \times\right.} \\
\vdots \\
{\left[A\left(\hat{\mathbf{q}}_{k}^{-}\right) \mathbf{r}_{N} \times\right.}\end{array}\right]\right|_{t_{k}}\end{array}$ \\
\hline Update & $\begin{array}{c}P_{k}^{r+}=\left(I_{6}-K_{k}^{r}\left[\begin{array}{ll}\tilde{H}_{k}\left(\hat{\mathbf{x}}_{k}^{r-}\right) & 0_{3 N \times 3}\end{array}\right]\right) P_{k}^{r-} \\
\Delta \hat{\mathbf{x}}_{k}^{r+\triangleq}\left[\begin{array}{c}\boldsymbol{\delta} \hat{\boldsymbol{\vartheta}}_{k}^{+} \\
\Delta \hat{\boldsymbol{\beta}}_{k}^{+}\end{array}\right]=K_{k}^{r}\left[\tilde{\mathbf{y}}_{k}-\mathbf{h}_{k}\left(\hat{\mathbf{x}}_{k}^{r-}\right)\right] \\
\mathbf{h}_{k}\left(\hat{\mathbf{x}}_{r_{k}}^{r-}\right)=\left.\left[\begin{array}{c}A\left(\hat{\mathbf{q}}_{k}^{-}\right) \mathbf{r}_{1} \\
\vdots \\
A\left(\hat{\mathbf{q}}_{k}^{-}\right) \mathbf{r}_{N}\end{array}\right]\right|_{t_{k}} \\
\hat{\mathbf{q}}^{*}=\hat{\mathbf{q}}_{k}^{-}+\frac{1}{2} \Xi\left(\hat{\mathbf{q}}_{k}^{-}\right) \boldsymbol{\delta} \hat{\boldsymbol{\vartheta}}_{k}^{+} \\
\hat{\mathbf{q}}_{k}^{+}=\hat{\mathbf{q}}^{*} /\left\|\hat{\mathbf{q}}^{*}\right\| \\
M^{L} \hat{\boldsymbol{\beta}}_{k}^{+}=M^{L} \hat{\boldsymbol{\beta}}_{k}^{-}+\Delta \hat{\boldsymbol{\beta}}_{k}^{+}\end{array}$ \\
\hline Propagation & $\begin{array}{c}\hat{\boldsymbol{\psi}}_{k+1, k}=M^{L} \tilde{\boldsymbol{\varphi}}_{k+1}-M^{L} \tilde{\boldsymbol{\varphi}}_{k}-M^{L} \hat{\boldsymbol{\beta}}_{k} \delta t \\
\hat{\mathbf{q}}_{k+1}=\exp \left(\frac{1}{2}\left[\hat{\boldsymbol{\psi}}_{k+1, k} \otimes\right]\right) \hat{\mathbf{q}}_{k} \\
M^{L} \hat{\boldsymbol{\beta}}_{k+1}=M^{L} \hat{\boldsymbol{\beta}}_{k} \\
P_{k+1}^{r}=\tilde{\Phi}\left(t_{k+1}, t_{k}\right) P_{k}^{r} \tilde{\Phi}^{T}\left(t_{k+1}, t_{k}\right)+Q^{r}\end{array}$ \\
\hline
\end{tabular}

where $\tilde{\alpha}_{i}$ and $\tilde{\beta}_{i}$ are focal plane measurements. Their respective true quantities are denoted by $\alpha_{i}$ and $\beta_{i}$. Defining the $2 \times 1$ vector $\gamma_{i} \triangleq\left[\begin{array}{ll}\alpha_{i} & \beta_{i}\end{array}\right]^{T}$, then the measurement model follows

$$
\tilde{\gamma}_{i}=\gamma_{i}+\mathbf{v}_{i}
$$

where $\mathbf{v}_{i}$ is a zero-mean Gaussian noise process. A frequently used covariance for $\mathbf{v}_{i}$ is given 

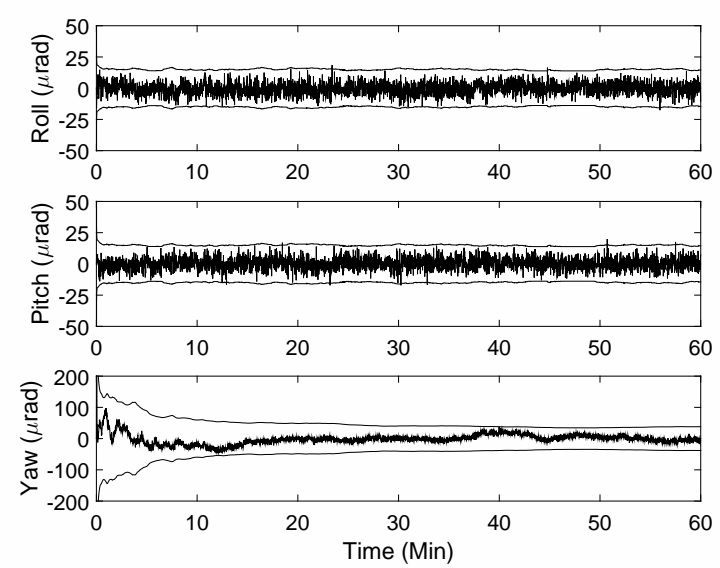

(a) Attitude-Estimate Errors
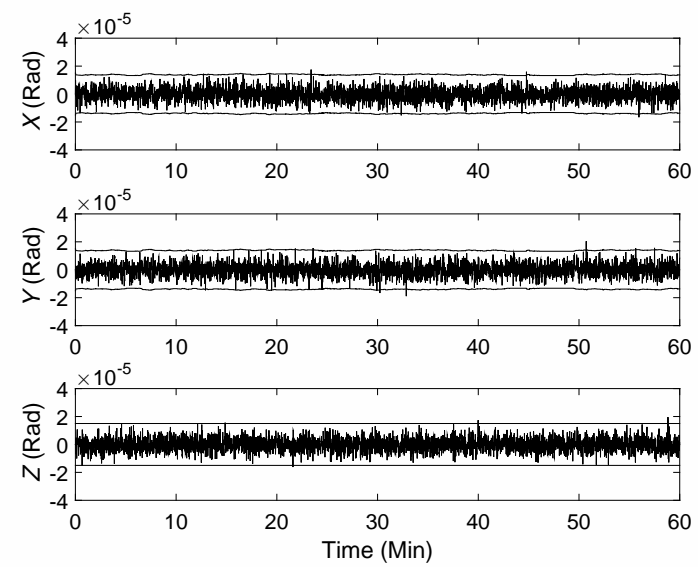

(c) RIG Angle-Estimate Errors
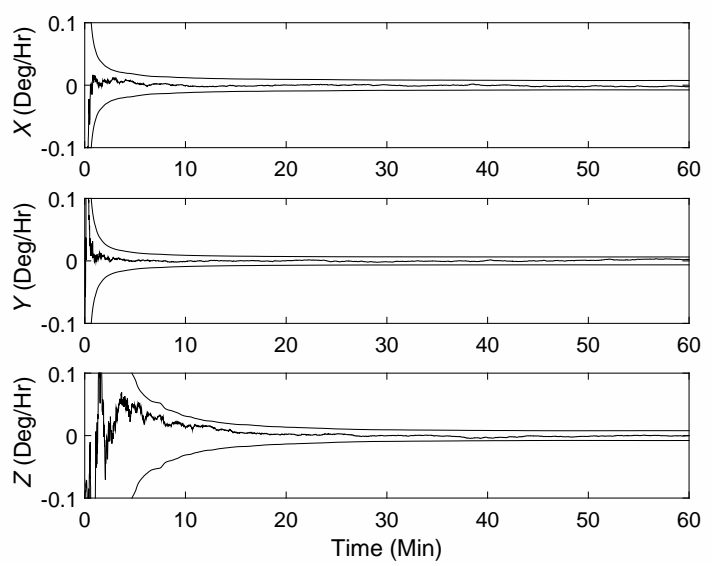

(b) Bias-Estimate Errors
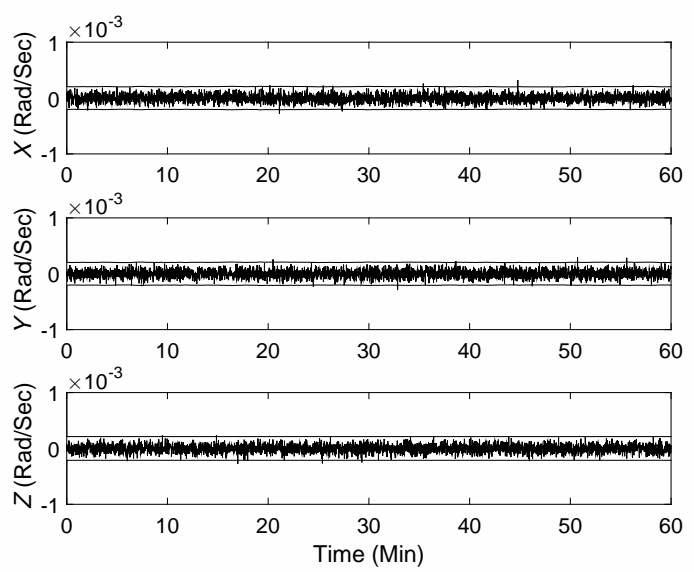

(d) Angular Velocity-Estimate Errors

Figure 2. RIG MEKF Errors and $3 \sigma$ Bounds

by $[16]$

$$
R_{i}^{\mathrm{FOCAL}}=\frac{\sigma^{2}}{1+d\left(\alpha_{i}^{2}+\beta_{i}^{2}\right)}\left[\begin{array}{cc}
\left(1+d \alpha_{i}^{2}\right)^{2} & \left(d \alpha_{i} \beta_{i}\right)^{2} \\
\left(d \alpha_{i} \beta_{i}\right)^{2} & \left(1+d \beta_{i}^{2}\right)^{2}
\end{array}\right]
$$

where $d$ is set to 1 and $\sigma=(0.005 / 3) \times(\pi / 180)$ rad. Note that Eqs. (104)-(106) are used to generate the simulated measurements, while Eq. (8) is used in the RIG MEKF, which approximates the actual covariance. The star tracker can sense up to 10 stars in a $6^{\circ} \times 6^{\circ}$ field-of-view, and the star catalog contains stars up to a magnitude of 6.0, the assumed star tracker sensitivity limit. The star tracker's boresight is defined by its corresponding sensor $z$-axis, which is assumed to be along the negative spacecraft body z-axis. Star images are 
taken at 1-second intervals. A plot of the number of available stars is shown in Figure 1.

The spacecraft is assumed to be equipped with three RIGs with their boresights along the spacecraft body axes. The noise parameters for each axis of the RIG measurements are equal with $Q_{e}=\sigma_{e}^{2} I_{3 \times 3}, Q_{u}=\sigma_{u}^{2} I_{3 \times 3}$, and $Q_{v}=\sigma_{v}^{2} I_{3 \times 3}$. The specific values for $\sigma_{e}, \sigma_{u}$, and $\sigma_{v}$ are $\sigma_{e}=5 \times 10^{-6} \mathrm{rad}, \sigma_{u}=\sqrt{10} \times 10^{-10} \mathrm{rad} / \mathrm{sec}^{3 / 2}$, and $\sigma_{v}=\sqrt{10} \times 10^{-7} \mathrm{rad} / \mathrm{sec}^{1 / 2}$. The initial bias for each axis is given by $0.1 \mathrm{deg} / \mathrm{hr}$. The RIG measurements are output every 0.1 seconds, i.e. 10 times faster than the star tracker measurements. The initial attitude estimate is given by its true value. The initial bias estimates are all set to zero, and the initial RIG angle estimates are set to their measured values. The initial error-covariance for the attitude-estimate matrix is isotropic with a $3 \sigma$ value of $1 \mathrm{deg}$. The error-covariance for the bias-estimate matrix is isotropic with a $3 \sigma$ value of $1 \mathrm{deg} / \mathrm{hr}$, and the error-covariance for the RIG-estimate matrix is isotropic with a variance of $\sigma_{e}^{2}$.

The results in Figure 2 show good filter convergence, consistent with results obtained using rate gyros. All errors are within their respective $3 \sigma$ bounds. Figure 2(a) shows how the attitude errors slightly increase at times when fewer stars are available, which is expected. The attitude $3 \sigma$ bounds for the off-boresight axes at steady-state are about $16 \mu \mathrm{rad}$. The single-axis case gives an analytical steady-state $3 \sigma$ bound of about $17 \mu \mathrm{rad}[7,13]$. The bias $3 \sigma$ bounds for the off-boresight axes at steady-state are about $6.4 \times 10^{-3} \mathrm{deg} / \mathrm{hr}$. The analytic steady-state single-axis $3 \sigma$ estimate is about $6.5 \times 10^{-3} \mathrm{deg} / \mathrm{hr}$. The RIG angle $3 \sigma$ bounds for the off-boresight axes at steady-state are about $1.5 \times 10^{-5} \mathrm{rad}$. The steadystate single-axis analysis gives a $3 \sigma$ bound of about $1.5 \times 10^{-5} \mathrm{rad}$. This shows how the steady-state single-axis results can be used to accurately assess the performance of the full three-axis case.

Results of the reduced-order RIG MEKF using the same simulation parameters are shown in Figure 3. The attitude errors in Figure 3(a) exhibit much more fluctuation due to the number of stars than the errors shown in Figure 2(a). The single-axis case gives a $3 \sigma$ bound of about $77 \mu \mathrm{rad}$, which is slightly larger than the average errors seem in Figure 3(a). The bias $3 \sigma$ bounds for the off-boresight axes at steady-state are about $0.26 \mathrm{deg} / \mathrm{hr}$. The single-axis case gives a $3 \sigma$ bound of about $0.27 \mathrm{deg} / \mathrm{hr}$. Good filter convergence is again seen. All errors are within their respective $3 \sigma$ bounds, which seems to show that although correlations are ignored in the reduced-order filter, the estimates themselves are at least consistent. However, the estimate errors are much larger using the reduced-order filter than the full-order filter. This is consistent with the single-axis analysis, which shows that ignoring the correlations in the measurements greatly overestimates the contributions of the RIG output noise.

Figure 4 shows the results of a third simulation using the standard MEKF filter that does not take the $\sigma_{e}$ term into account $[7,11]$. The RIG measurements are simulated using the same gyro noise parameters as the other simulations: $\sigma_{e}=5 \times 10^{-6} \mathrm{rad}, \sigma_{u}=\sqrt{10} \times 10^{-10}$ 

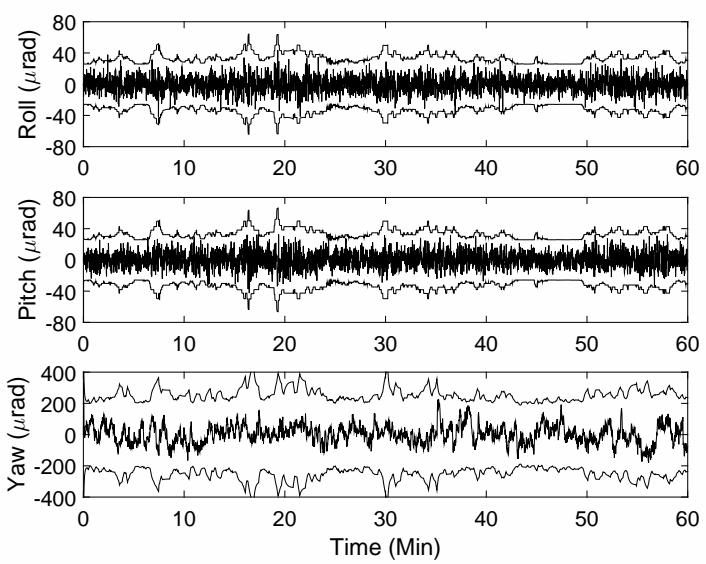

(a) Attitude-Estimate Errors
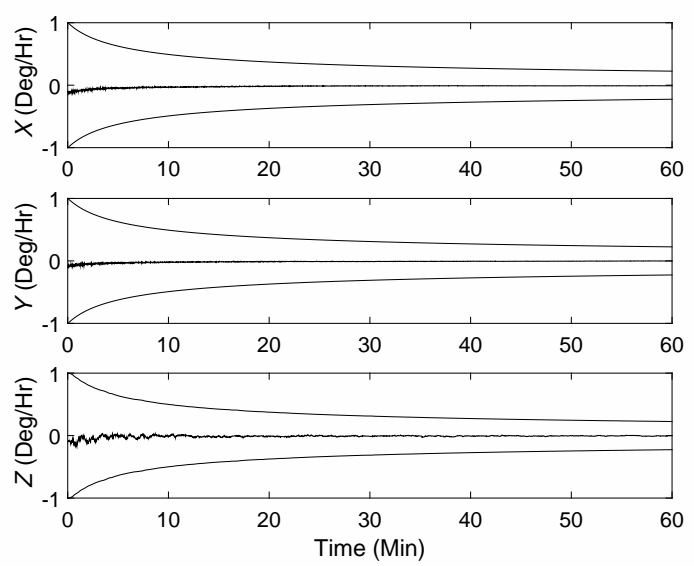

(b) Bias-Estimate Errors
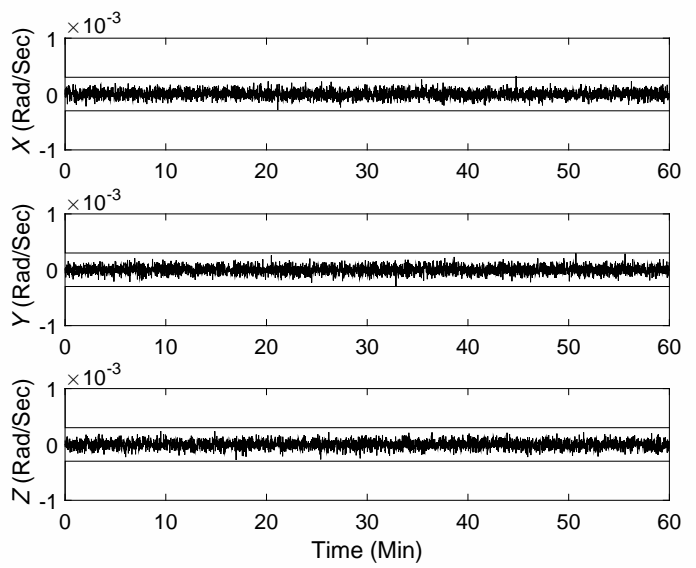

(c) Angular Velocity-Estimate Errors

Figure 3. Reduced-Order RIG MEKF Errors and $3 \sigma$ Bounds

$\mathrm{rad} / \mathrm{sec}^{3 / 2}$, and $\sigma_{v}=\sqrt{10} \times 10^{-7} \mathrm{rad} / \mathrm{sec}^{1 / 2}$. A finite difference of the RIG angles is taken to produce angular rate observations. This filter is equivalent to the reduced filter of Table 2 using RIG outputs while setting $\sigma_{e}=0$ in the filter. The bias-estimate errors and their $3 \sigma$ bounds agree very closely with the results plotted in Figure 1, except for some initial transients. The attitude errors on all three axes and the $3 \sigma$ bounds on the star tracker's boresight axis also agree after initial transients have died out, but the standard MEKF filter underestimates the variance of the attitude errors on the other two axes. This shows that naively ignoring a nonzero value of $\sigma_{e}$ may produce inconsistent estimates. Comparison of Figures 3 and 4 shows, though, that completely ignoring the RIG measurement output noise can be preferable to including it in a filter that does not augment the state vector with RIG 
angle parameters.
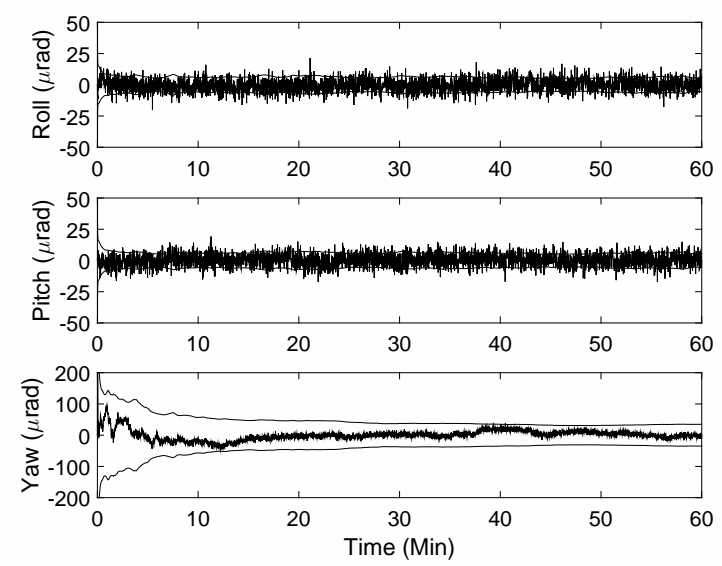

(a) Attitude-Estimate Errors
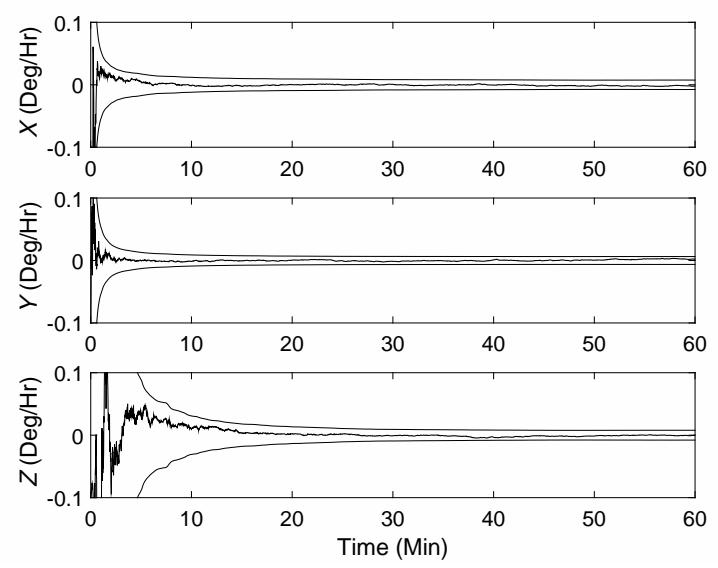

(b) Bias-Estimate Errors

Figure 4. Standard MEKF Attitude Estimator Results

\section{Conclusions}

This paper presents two filters for attitude estimation that incorporate rate-integrating gyros. The first filter uses an augmented state approach that accounts for correlations between contributions of the rate-integrating gyro output noise to the angle variance, while the second one ignores these correlations. Simulation results involving a star tracker coupled with rate-integrating gyros in a multiplicative extended Kalman framework validate that both filters are consistent estimators, but that ignoring the correlations results in significantly larger attitude estimation errors. This is consistent with analytical expressions for the singleaxis case, which show that ignoring these correlations overestimates the gyro output noise contribution to the process noise covariance. These results are also compared with a rategyro-based filter using finite differences of rate-integrating gyro outputs and ignoring the measurement noise in these outputs. This comparison shows that naively ignoring the output noise can produce acceptable attitude and bias estimates if it is not excessively large, but the filter may be inconsistent in producing erroneously small estimates of its errors. The approach shown in this paper can easily be extended to other applications, such as inertial navigation using rate-integrating gyros, by simply appending the state vector to estimate other states, such as position, velocity, and accelerometer biases. 


\section{Appendix: Modeling Rate-Integrating Gyro Noise}

Equations to model RIG noise in the single-axis case are derived here. These can be used in the three-axis case under the usual assumption that the matrices $Q_{e}, Q_{u}$ and $Q_{v}$ are diagonal. In the gyro model shown in Ref. [14] the bias $\beta$ and angle output $\varphi$ of a RIG obey

$$
\begin{gathered}
\beta_{k+1}=\beta_{k}+\int_{t_{k}}^{t_{k+1}} \eta_{u}(\tau) d \tau \\
\varphi_{k+1}=\varphi_{k}+\int_{t_{k}}^{t_{k+1}} \tilde{\omega}(\tau) d \tau=\varphi_{k}+\int_{t_{k}}^{t_{k+1}}\left[\omega(\tau)+\beta(\tau)+\eta_{v}(\tau)\right] d \tau \\
=\bar{\varphi}+\int_{t_{k}}^{t_{k+1}} \int_{t_{k}}^{\tau} \eta_{u}\left(\tau^{\prime \prime}\right) d \tau^{\prime \prime} d \tau+\int_{t_{k}}^{t_{k+1}} \eta_{v}(\tau) d \tau
\end{gathered}
$$

where

$$
\bar{\varphi} \triangleq \varphi_{k}+\beta_{k} \delta t+\int_{t_{k}}^{t_{k+1}} \omega(\tau) d \tau
$$

The zero-mean processes $\eta_{v}$ and $\eta_{u}$ have autocorrelations $E\left\{\eta_{v}(t) \eta_{v}(\tau)\right\}=\sigma_{v}^{2} \delta(t-\tau)$, $E\left\{\eta_{u}(t) \eta_{u}(\tau)\right\}=\sigma_{u}^{2} \delta(t-\tau)$, respectively, and $E\left\{\eta_{v}(t) \eta_{u}(\tau)\right\}=0$. To obtain the correct means, the modeled quantities, indicated by the subscript $m$, must be given by

$$
\begin{aligned}
\varphi_{m_{k+1}} & =\varphi_{m_{k}}+\beta_{m_{k}} \delta t+\int_{t_{k}}^{t_{k+1}} \omega(\tau) d \tau+\text { zero-mean random number } \\
& =\bar{\varphi}_{m}+\text { zero-mean random number } \\
\beta_{m_{k+1}} & =\beta_{m_{k}}+\text { zero-mean random number }
\end{aligned}
$$

where the random numbers, which turn out to be correlated, must be chosen so that the second-order statistics of the modeled quantities agree with those of the true equations. The autocorrelation of the RIG drift bias is

$$
\begin{aligned}
E\left\{\beta_{k+1}^{2}\right\} & =E\left\{\left[\beta_{k}+\int_{t_{k}}^{t_{k+1}} \eta_{u}(\tau) d \tau\right]\left[\beta_{k}+\int_{t_{k}}^{t_{k+1}} \eta_{u}\left(\tau^{\prime}\right) d \tau^{\prime}\right]\right\} \\
& =E\left\{\beta_{k}^{2}\right\}+\sigma_{u}^{2} \int_{t_{k}}^{t_{k+1}} \int_{t_{k}}^{t_{k+1}} \delta\left(\tau-\tau^{\prime}\right) d \tau^{\prime} d \tau=E\left\{\beta_{k}^{2}\right\}+\sigma_{u}^{2} \delta t
\end{aligned}
$$

Therefore, RIG drift bias can be modeled by

$$
\beta_{m_{k+1}}=\beta_{m_{k}}+\sigma_{u} \delta t^{1 / 2} \mathcal{N}_{u}
$$


where $\mathcal{N}_{u}$ is a zero-mean random number with unit variance. The correlation of the drift bias and angle is given by

$$
\begin{aligned}
E\left\{\beta_{k+1} \varphi_{k+1}\right\} & =E\left\{\left[\beta_{k}+\int_{t_{k}}^{t_{k+1}} \eta_{u}\left(\tau^{\prime}\right) d \tau^{\prime}\right]\right. \\
& \left.\times\left[\bar{\varphi}+\int_{t_{k}}^{t_{k+1}} \int_{t_{k}}^{\tau} \eta_{u}\left(\tau^{\prime \prime}\right) d \tau^{\prime \prime} d \tau+\int_{t_{k}}^{t_{k+1}} \eta_{v}(\tau) d \tau\right]\right\} \\
& =E\left\{\beta_{k} \bar{\varphi}\right\}+\sigma_{u}^{2} \int_{t_{k}}^{t_{k+1}} \int_{t_{k}}^{t_{k+1}} \int_{t_{k}}^{\tau} \delta\left(\tau^{\prime}-\tau^{\prime \prime}\right) d \tau^{\prime \prime} d \tau^{\prime} d \tau \\
& =E\left\{\beta_{k} \bar{\varphi}\right\}+\sigma_{u}^{2} \int_{t_{k}}^{t_{k+1}}\left(\tau-t_{k}\right) d \tau=E\left\{\beta_{k} \bar{\varphi}\right\}+\frac{1}{2} \sigma_{u}^{2} \delta t^{2}
\end{aligned}
$$

This equation is satisfied by modeling the RIG output as

$$
\begin{aligned}
\varphi_{m_{k+1}} & =\bar{\varphi}_{m_{k}}+\frac{1}{2} \sigma_{u} \delta t^{3 / 2} \mathcal{N}_{u}+c \mathcal{N}_{v} \\
& =\varphi_{m_{k}}+\frac{1}{2}\left[\beta_{m_{k+1}}+\beta_{m_{k}}\right] \delta t+\int_{t_{k}}^{t_{k+1}} \omega(\tau) d \tau+c \mathcal{N}_{v}
\end{aligned}
$$

where $c$ is a constant to be determined, and $\mathcal{N}_{v}$ is a zero-mean, unit-variance random number uncorrelated with $\mathcal{N}_{u}$. To evaluate $c$ the autocorrelation of the RIG angle output is computed:

$$
\begin{aligned}
E\left\{\varphi_{k+1}^{2}\right\} & =E\left\{\left[\bar{\varphi}+\int_{t_{k}}^{t_{k+1}} \int_{t_{k}}^{\tau^{\prime}} \eta_{u}\left(\tau^{\prime \prime \prime}\right) d \tau^{\prime \prime \prime} d \tau^{\prime}+\int_{t_{k}}^{t_{k+1}} \eta_{v}\left(\tau^{\prime}\right) d \tau^{\prime}\right]\right. \\
& \left.\times\left[\bar{\varphi}+\int_{t_{k}}^{t_{k+1}} \int_{t_{k}}^{\tau} \eta_{u}\left(\tau^{\prime \prime}\right) d \tau^{\prime \prime} d \tau+\int_{t_{k}}^{t_{k+1}} \eta_{v}(\tau) d \tau\right]\right\} \\
& =E\left\{\bar{\varphi}^{2}\right\}+\sigma_{u}^{2} \int_{t_{k}}^{t_{k+1}} \int_{t_{k}}^{t_{k+1}} \int_{t_{k}}^{\tau} \int_{t_{k}}^{\tau^{\prime}} \delta\left(\tau^{\prime \prime \prime}-\tau^{\prime}\right) d \tau^{\prime \prime \prime} d \tau^{\prime \prime} d \tau^{\prime} d \tau \\
& +\sigma_{v}^{2} \int_{t_{k}}^{t_{k+1}} \int_{t_{k}}^{t_{k+1}} \delta\left(\tau-\tau^{\prime}\right) d \tau^{\prime} d \tau
\end{aligned}
$$

Now

$$
\begin{gathered}
\int_{t_{k}}^{t_{k+1}} \int_{t_{k}}^{t_{k+1}} \int_{t_{k}}^{\tau} \int_{t_{k}}^{\tau^{\prime}} \delta\left(\tau^{\prime \prime \prime}-\tau^{\prime}\right) d \tau^{\prime \prime \prime} d \tau^{\prime \prime} d \tau^{\prime} d \tau=\int_{t_{k}}^{t_{k+1}} \int_{t_{k}}^{t_{k+1}} \min \left(\tau^{\prime}-t_{k}, \tau-t_{k}\right) d \tau^{\prime} d \tau \\
=\int_{0}^{\delta t} \int_{0}^{\delta t} \min (x, y) d x d y=\int_{0}^{\delta t}\left(\int_{0}^{y} x d x+\int_{y}^{\delta t} y d x\right) d y \\
=\int_{0}^{\delta t}\left[\frac{1}{2} y^{2}+y(\delta t-y)\right] d y=\frac{1}{3} \delta t^{3}
\end{gathered}
$$


and the $\sigma_{v}^{2}$ integral is the same as the $\sigma_{u}^{2}$ integral in Eq. (A.4). Therefore, Eq. (A.8) becomes

$$
E\left\{\varphi_{k+1}^{2}\right\}=E\left\{\bar{\varphi}^{2}\right\}+\frac{1}{3} \sigma_{u}^{2} \delta t^{3}+\sigma_{v}^{2} \delta t=E\left\{\bar{\varphi}^{2}\right\}+\frac{1}{4} \sigma_{u}^{2} \delta t^{3}+c^{2}
$$

Since $E\left\{\bar{\varphi}^{2}\right\}=E\left\{\bar{\varphi}_{m}^{2}\right\}$ by construction, this means that

$$
c=\left(\sigma_{v}^{2} \delta t+\frac{1}{12} \sigma_{u}^{2} \delta t^{3}\right)^{1 / 2}
$$

Then Eq. (A.7) simply becomes

$$
\varphi_{m_{k+1}}=\varphi_{m_{k}}+\frac{1}{2}\left[\beta_{m_{k+1}}+\beta_{m_{k}}\right] \delta t+\int_{t_{k}}^{t_{k+1}} \omega(\tau) d \tau+\left(\sigma_{v}^{2} \delta t+\frac{1}{12} \sigma_{u}^{2} \delta t^{3}\right)^{1 / 2} \mathcal{N}_{v}
$$

The modeled measurement is then given by $\tilde{\varphi}_{m_{k}}=\varphi_{m_{k}}+v_{e}$, where $v_{e}$ is a zero-mean Gaussian white-noise process with variance $\sigma_{e}^{2}$.

The variance of $\tilde{\varphi}_{m_{k+1}}$ is now derived. Substituting Eq. (A.5) into Eq. (A.12), and using $\tilde{\varphi}_{m_{k+1}}=\varphi_{m_{k+1}}+v_{e}$ leads to

$$
\begin{aligned}
\tilde{\varphi}_{m_{k+1}} & =\varphi_{m_{k}}+\beta_{m_{k}} \delta t+\int_{t_{k}}^{t_{k+1}} \omega(\tau) d \tau+\frac{1}{2} \sigma_{u} \delta t^{3 / 2} \mathcal{N}_{u} \\
& +\left(\sigma_{v}^{2} \delta t+\frac{1}{12} \sigma_{u}^{2} \delta t^{3}\right)^{1 / 2} \mathcal{N}_{v}+v_{e}
\end{aligned}
$$

The expectation of this equation given $\varphi_{m_{k}}$ and $\beta_{m_{k}}$ is

$$
E\left\{\tilde{\varphi}_{m_{k+1}} \mid \varphi_{m_{k}}, \beta_{m_{k}}\right\}=\varphi_{m_{k}}+\beta_{m_{k}} \delta t+\int_{t_{k}}^{t_{k+1}} \omega(\tau) d \tau
$$

The variance, denoted by $R_{m_{k+1}}$, is now computed through

$$
R_{m_{k+1}}=E\left\{\left(\frac{1}{2} \sigma_{u} \delta t^{3 / 2} \mathcal{N}_{u}\right)^{2}\right\}+\left(\sigma_{v}^{2} \delta t+\frac{1}{12} \sigma_{u}^{2} \delta t^{3}\right) E\left\{\mathcal{N}_{v}^{2}\right\}+E\left\{v_{e}^{2}\right\}
$$

Taking the expectations and collecting terms gives

$$
R_{m_{k+1}}=\sigma_{v}^{2} \delta t+\frac{1}{3} \sigma_{u}^{2} \delta t^{3}+\sigma_{e}^{2}
$$

Note that this is equivalent to the upper left corner of $Q(\delta t)$ in Eq. (24). 


\section{References}

${ }^{1}$ Crassidis, J. L., Markley, F. L., and Cheng, Y., "Survey of Nonlinear Attitude Estimation Methods," Journal of Guidance, Control, and Dynamics, Vol. 30, No. 1, Jan.-Feb. 2007, pp. 12-28, doi:10.2514/1.22452.

${ }^{2}$ Savage, P. G., "Strapdown Inertial Navigation Integration Algorithm Design Part 2: Velocity and Position Algorithms," Journal of Guidance, Control, and Dynamics, Vol. 21, No. 2, March-April 1998, pp. 208-221, doi:10.2514/1.22452.

${ }^{3}$ Kinsey, J. C., Eustice, R. M., and Whitcomb, L. L., "A Survey of Underwater Vehicle Navigation: Recent Advances and New Challenges," Proceedings of the IFAC Conference of Manoeuvring and Control of Marine Craft, Lisbon, Portugal, Sept. 2006, Invited paper.

${ }^{4}$ Barshan, B. and Durrant-Whyte, H. F., "Inertial Navigation Systems for Mobile Robots," IEEE Transactions on Robotics and Automation, Vol. 11, No. 3, June 1995, pp. 328-342, doi:10.1109/70.388775.

${ }^{5}$ Fallah, N., Apostolopoulos, I., Bekris, K., and Folmer, E., "Indoor Human Navigation Systems: A Survey," Interacting with Computers, Vol. 25, No. 1, Feb. 2013, pp. 21-33, doi:10.1093/iwc/iws010.

${ }^{6}$ Fallon, L., "Gyroscopes," Spacecraft Attitude Determination and Control, edited by J. R. Wertz, chap. 6.5, Kluwer Academic Publishers, The Netherlands, 1978, doi:10.1007/978-94-009-9907-7.

${ }^{7}$ Markley, F. L. and Crassidis, J. L., Fundamentals of Spacecraft Attitude Determination and Control, Springer, New York, NY, 2014, pp. 46, 257-260, 263-269, doi:10.1007/978-1-4939-0802-8.

${ }^{8}$ Senkal, D., Ng, E. J., Hong, V., Yang, Y., Ahn, C. H., Kenny, T. W., and Shkel, A. M., "Parametric Drive of a Toroidal MEMS Rate Integrating Gyroscope Demonstrating < 20 PPM Scale Factor Stability," 28th IEEE International Conference on Micro Electro Mechanical Systems (MEMS), Estoril, Portugal, Jan. 2015, pp. 29-32, doi:10.1109/MEMSYS.2015.7050878.

${ }^{9}$ Pittelkau, M. E., "Sensors for Attitude Determination," Encyclopedia of Aerospace Engineering, edited by R. Blockley and W. Shyy, John Wiley \& Sons, Inc., Chichester, UK, 2010.

${ }^{10}$ King, A. D., "Inertial Navigation - Forty Years of Evolution," GEC Review, Vol. 13, No. 3, 1998, pp. 140-149.

${ }^{11}$ Lefferts, E. J., Markley, F. L., and Shuster, M. D., "Kalman Filtering for Spacecraft Attitude Estimation," Journal of Guidance, Control, and Dynamics, Vol. 5, No. 5, Sept.-Oct. 1982, pp. 417-429, doi:10.2514/3.56190.

${ }^{12}$ Unhelkar, V. V. and Hablani, H. B., "Satellite Attitude Determination with Attitude Sensors and Gyros Using Steady-State Kalman Filter," Advances in Estimation, Navigation, and Spacecraft Control: Selected Papers of the Itzhack Y. Bar-Itzhack Memorial Symposium on Estimation, Navigation, and Spacecraft Control, edited by D. Choukroun, Y. Oshman, J. Thienel, and M. Idan, Springer-Verlag, Berlin, Germany, 2010, pp. 413-438, doi:10.1007/978-3-662-44785-7.

${ }^{13}$ Markley, F. L. and Reynolds, R. G., "Analytic Steady-State Accuracy of a Spacecraft Attitude Estimator," Journal of Guidance, Control, and Dynamics, Vol. 23, No. 6, Nov.-Dec. 2000, pp. 1065-1067, doi:10.2514/2.4648.

${ }^{14}$ Farrenkopf, R. L., "Analytic Steady-State Accuracy Solutions for Two Common Spacecraft Attitude Estimators," Journal of Guidance and Control, Vol. 1, No. 4, July-Aug. 1978, pp. 282-284, doi:10.2514/3.55779.

${ }^{15}$ Shuster, M. D., "A Survey of Attitude Representations," The Journal of the Astronautical Sciences, Vol. 41, No. 4, Oct.-Dec. 1993, pp. 439-517. 
${ }^{16}$ Shuster, M. D., "Kalman Filtering of Spacecraft Attitude and the QUEST Model," The Journal of the Astronautical Sciences, Vol. 38, No. 3, July-Sept. 1990, pp. 377-393.

${ }^{17}$ Shuster, M. D. and Oh, S. D., "Attitude Determination from Vector Observations," Journal of Guidance and Control, Vol. 4, No. 1, Jan.-Feb. 1981, pp. 70-77, doi:10.2514/3.19717.

${ }^{18}$ Markley, F. L., "Attitude Estimation or Quaternion Estimation?" The Journal of the Astronautical Sciences, Vol. 52, No. 1 \& 2, Jan.-June 2004, pp. 221-238.

${ }^{19}$ Cheng, Y., Crassidis, J. L., and Markley, F. L., "Attitude Estimation for Large Field-of-View Sensors," The Journal of the Astronautical Sciences, Vol. 54, No. 3/4, July-Dec. 2006, pp. 433-448, doi:10.1007/BF03256499.

${ }^{20}$ Crassidis, J. L. and Junkins, J. L., Optimal Estimation of Dynamic Systems, chap. 3, CRC Press, Boca Raton, FL, 2nd ed., 2012.

${ }^{21}$ Shuster, M. D., "Maximum Likelihood Estimation of Spacecraft Attitude," The Journal of the Astronautical Sciences, Vol. 37, No. 1, Jan.-March 1989, pp. 79-88. 